\title{
Emergence of magnetic flux from the convection zone into the corona
}

\author{
V. Archontis ${ }^{1}$, F. Moreno-Insertis ${ }^{1,2}$, K. Galsgaard ${ }^{3,4}$, A. Hood ${ }^{3}$, and E. O'Shea ${ }^{1}$ \\ ${ }^{1}$ Instituto de Astrofisica de Canarias (IAC), La Laguna (Tenerife), Spain \\ e-mail: vasilis@ll.iac.es \\ 2 Department of Astrophysics, Faculty of Physics, Universidad de La Laguna, La Laguna (Tenerife), Spain \\ 3 School of Mathematics and Statistics, University of St Andrews, UK \\ ${ }^{4}$ Niels Bohr Institute for Astronomy, Physics and Geophysics, Copenhagen, Denmark
}

Received 23 December 2003 / Accepted 21 April 2004

\begin{abstract}
Numerical experiments of the emergence of magnetic flux from the uppermost layers of the solar interior to the photosphere and its further eruption into the low atmosphere and corona are carried out. We use idealized models for the initial stratification and magnetic field distribution below the photosphere similar to those used for multidimensional flux emergence experiments in the literature. The energy equation is adiabatic except for the inclusion of ohmic and viscous dissipation terms, which, however, become important only at interfaces and reconnection sites. Three-dimensional experiments for the eruption of magnetic flux both into an unmagnetized corona and into a corona with a preexisting ambient horizontal field are presented. The shocks preceding the rising plasma present the classical structure of nonlinear Lamb waves. The expansion of the matter when rising into the atmosphere takes place preferentially in the horizontal directions: a flattened (or oval) low plasma- $\beta$ ball ensues, in which the field lines describe loops in the corona with increasing inclination away from the vertical as one goes toward the sides of the structure. Magnetograms and velocity field distributions on horizontal planes are presented simultaneously for the solar interior and various levels in the atmosphere. Since the background pressure and density drop over many orders of magnitude with increasing height, the adiabatic expansion of the rising plasma yields very low temperatures. To avoid this, the entropy of the rising fluid elements should be increased to the high values of the original atmosphere via heating mechanisms not included in the present numerical experiments.

The eruption of magnetic flux into a corona with a preexisting magnetic field pointing in the horizontal direction yields a clear case of essentially three-dimensional reconnection when the upcoming and ambient field systems come into contact. The coronal ambient field is chosen at time $t=0$ perpendicular to the direction of the tube axis and thus, given the twist of the magnetic tube, almost anti-parallel to the field lines at the upper boundary of the rising plasma ball. A thin, dome-shaped current layer is formed at the interface between the two flux systems, in which ohmic dissipation and heating are taking place. The reconnection proceeds by merging successive layers on both sides of the reconnection site; however, this occurs not only at the cusp of the interface, but, also, gradually along its sides in the direction transverse to the ambient magnetic field. The topology of the magnetic field in the atmosphere is thereby modified: the reconnected field lines typically are part of the flanks of the tube below the photosphere but then join the ambient field system in the corona and reach the boundaries of the domain as horizontal field lines.
\end{abstract}

Key words. Sun: corona - Sun: magnetic fields - Sun: interior - magnetohydrodynamics (MHD) - methods: numerical stars: activity

\section{Introduction}

The evolution of the solar corona is controlled by the local magnetic field. However, the coronal magnetic field can be profoundly modified in response to the emergence of magnetic flux from below the photosphere in active regions. Largescale emerging field is generated in the solar interior, probably in the dynamo region around the bottom of the convection zone. The dynamo effect may also produce field structures on smaller scales, especially closer to the surface, leading to localized eruption processes as in, e.g., ephemeral active regions.
In either case, the emergence of new magnetic fields can destabilize the existing field structures and cause a variety of largescale dynamic coronal phenomena. Prominence eruptions, solar flares and Coronal Mass Ejections (CMEs) are just a few.

Observations of large scale magnetic fields emerging into the corona have been identified by the TRACE and SOHO satellites. The coronal fields are normally outlined by plasma emitting in EUV or X-rays (e.g. TRACE and EIT). For the photosphere, direct measurements of the magnetic field, both by satellite instruments (like MDI) as well as ground-based detectors, yield a wealth of information. Vector 
magnetograms, in particular, permit the reconstruction of all three components of the magnetic field. Shortly after the appearance of flux at the photosphere, a system of bright coronal loops appears in the EUV and X-ray detectors. These coronal loops seem to join the opposite polarities of the active regions and probably delineate magnetic field lines.

A necessary step for progress is to understand how the magnetic fields make their way up through the convection zone and emerge through the photosphere and chromosphere into the corona. In the deep convection zone, the magnetic field is expected to become unstable to buoyant instabilities and, thus, rise from the storage region to the photosphere throughout the solar cycle. The emergence of individual thin magnetic tubes across the convection zone has been the subject of active investigation during the past two decades, both analytically and numerically, in most cases modelling the magnetic tube as a $1 \mathrm{D}$ deformable continuum moving in the solar convection zone (Moreno-Insertis 1986, 1997; Choudhuri 1987; D’Silva \& Choudhuri 1993; Moreno-Insertis et al. 1994; Fan et al. 1994; Caligari et al. 1995). However, once the field reaches levels close to the solar surface, the magnetic tubes are no longer thin as regards the local prevailing length scales, and a 1D approximation cannot be applied. Their further rise across the photosphere and into the higher levels of the atmosphere is still a largely unexplored research domain.

It is the aim of this paper to analyse and illustrate some of the fundamental physical processes involved in the emergence of magnetic fields through a series of 3D MHD simulations. In the past years, there has been much interest in the literature in the eruption of magnetic flux through the photosphere to the corona. Yet, given the complexity of this process and the difficulty of discovering and explaining the basic phenomena taking place in multidimensional numerical experiments, there are still large gaps in the understanding of the physics involved. Numerical experimentation of this kind stretches back to the late eighties. A first batch of models were two-dimensional and dealt with the emergence of a horizontal flux sheet through the development of a buoyant instability with $\boldsymbol{k} \| \boldsymbol{B}$ (e.g., Shibata et al. 1989; 1992; Nozawa et al. 1992, and references therein). In most cases, the initial stratification consisted of two unmagnetized isothermal domains (simulating photosphere and corona), with a convection zone underneath, which had either an adiabatic or a superadiabatic temperature gradient. An interesting feature presented in those papers was the appearance of self-similar behavior in the nonlinear phase of the unstable evolution. They also calculated eigenfunctions for the linear stability problem associated with the particular initial condition they were using. Shibata et al. (1992), while still 2D, included an ambient field in the corona which was antiparallel to the field rising from the bottom of the integration box. Another class of papers was also two-dimensional (or, rather, 2.5 dimensional) but considered the rise of twisted magnetic flux tubes with traslational symmetry of all variables along their axis (Krall et al. 1998; Magara 2001), thereby extending to the atmosphere the 2.5D magnetic tube rise calculations of Moreno-Insertis \& Emonet (1996) and Emonet \& Moreno-Insertis (1998). Krall et al. (1998), in particular, included an ambient field oriented vertically which could help lower the minimum value of twist shown by Moreno-Insertis \& Emonet (1996) to be necessary to maintain the unity of the rising tube.

Three-dimensional calculations of the emergence of magnetic flux through the photosphere into the corona have been carried out by Matsumoto \& Shibata (1992), Matsumoto et al. (1993, 1998), Kusano et al. (1998), Fan (2001), Magara \& Longcope (2001, 2003), and Abbett \& Fisher (2003). In spite of the low resolution of the early simulation of Matsumoto et al. (1993), it yielded a number of important features of the process of 3D emergence. These authors studied the interchange $(\boldsymbol{k} \perp \boldsymbol{B})$ and quasi-interchange $\left(\boldsymbol{k} \| \boldsymbol{B}\right.$ with $\left.k H_{\mathrm{p}} \ll 1\right)$ instabilities in a flux sheet and in a magnetic tube in the photosphere, using as initial stratification two isothermal domains in height (photosphere, corona) and not including any convection zone below them. They obtained fast downflows within the flanks of the rising loops, with shocks at their feet, marked horizontal expansion in the higher atmospheric levels, and quasi self-similar expansion of the magnetic loops. Matsumoto et al. (1998), Fan (2001) and Magara \& Longcope $(2001,2003)$ have all carried out simulations including, from bottom to top, a convection zone, an isothermal photosphere and an isothermal corona. They suggest similarities between their results and different structures observed in the actual Sun, like sequences of S-shaped active regions observed in X-rays (Matsumoto et al. 1998), photospheric observations of the birth of active regions (Fan 2001) and X-ray sigmoids (Magara \& Longcope 2001). Magara \& Longcope (2003) study the force distribution along selected field lines of the rising plasma, concluding the importance of the magnetic pressure gradient to bring about the eruption. They also describe in detail the injection of magnetic energy and helicity into the atmosphere following the flux emergence process and discuss the relative contributions of horizontal shear motions and vertical flux at the photosphere for both quantities. Abbett \& Fisher (2003), finally, carry out the time integration separately for the convection zone and the atmosphere, with coupling between them prescribed by using as input for the upper domain the distribution of physical variables at a level close to the top of the convection zone.

Against the background of the literature on this topic, our objective in the present paper is twofold. Firstly, we carry out a numerical experiment with initial conditions similar to those used in most of the 3D simulations (initial magnetic tube below the surface, atmosphere consisting of two isothermal stretches joined by a steep temperature gradient). Like those simulations, we do not have radiative transfer or explicit coronal heating or cooling in the calculation. Yet, we include ohmic and viscous dissipation. We find and analyse various basic aspects of the emergence process which have not been described, or only insufficiently, in the literature. Among them we find: the propagation of shocks upward in the atmosphere preceding the emerging region; the instability criterion for the buoyant instability development when the magnetic tube reaches the photosphere; the low temperatures and high densities of the plasma rising in the atmosphere if one ignores all radiative or other thermal exchange processes in it; the large predominance of horizontal over vertical expansion of the emerging plasma in the transition region and corona, in an analogous manner to the shape of a cake overflowing its mould as it bakes (Fig. 5); 
the large downflows outside the erupting region which seem to fit the velocities observed in transition region and corona; the behavior of magnetic and velocity fields simultaneously at different heights from convection zone to corona, shown via synchronous magnetograms and velocity vector distributions on horizontal planes; etc.

Secondly, we perform a numerical experiment of a magnetic tube rising into an atmosphere which is uniformly magnetized upward of some $1500 \mathrm{~km}$ above the photosphere. The objective of this experiment is to have a first impression of the interaction between the upcoming and existing flux systems. For the configuration used here, with the ambient and upcoming magnetic fields pointing in opposite directions, we find that the two systems reconnect efficiently. This process occurs gradually as further plasma reaches coronal heights. A drastic change of the overall topology of the field ensues: the ambient coronal field lines starting from one side of the box get connected to one of the flanks of the rising tube, those starting from the other side, in turn, getting linked to the other flank of the tube. The rising plasma, therefore, makes its way into the higher levels through reconnection with the ambient field. As a result, in solar terms, the polarities of the new active region get connected to external magnetic domains (a feature repeatedly observed in satellite images of the eruption of an active region).

The layout of the paper is as follows: Sect. 2 presents the equations and the numerical code used in the experiments. Section 3 is devoted to a discussion of the initial condition used, both the background stratification (3.1) and the initial field distribution in the magnetic tube (3.2). The results of the experiment with a field-free background atmosphere are presented in Sects. 4 through 6. Section 7 describes the experiment with a pre-existing ambient magnetic field in higher levels of the atmosphere and presents the proof for ongoing reconnection, changes of connectivity and associated phenomena. Section 8 , finally contains a discussion and summary of conclusions.

\section{Equations and numerical approach}

\subsection{Equations}

For the experiments described in this paper, the threedimensional time-dependent resistive MHD equations are solved numerically. For this purpose the MHD equations are written in the form,

$$
\begin{aligned}
& \frac{\partial \rho}{\partial t}=-\nabla \cdot(\rho \boldsymbol{u}), \\
& \frac{\partial(\rho \boldsymbol{u})}{\partial t}=-\nabla \cdot(\rho \boldsymbol{u} \otimes \boldsymbol{u}+\underline{\tau})-\nabla p+\rho \boldsymbol{g}+\frac{\boldsymbol{J}}{c} \times \boldsymbol{B}, \\
& \frac{\partial e}{\partial t}=-\nabla \cdot(e \boldsymbol{u})-p \nabla \cdot \boldsymbol{u}+Q_{\text {Joule }}+Q_{\mathrm{visc}}, \\
& \frac{\partial \boldsymbol{B}}{\partial t}=-c \nabla \times \boldsymbol{E}, \\
& \boldsymbol{E}=-\frac{\boldsymbol{u}}{c} \times \boldsymbol{B}+\eta \frac{\boldsymbol{J}}{c^{2}}, \\
& \boldsymbol{J}=\frac{c}{4 \pi} \nabla \times \boldsymbol{B}, \\
& p=\rho T \frac{\mathcal{R}}{\tilde{\mu}},
\end{aligned}
$$

with density $\rho$, velocity $\boldsymbol{u}$, acceleration of gravity $\boldsymbol{g}=-g \boldsymbol{e}_{z}$, thermal energy per unit volume $e$, average atomic mass per particle $\tilde{\mu}$, magnetic field $\boldsymbol{B}$, electric field $\boldsymbol{E}$, magnetic resistivity $\eta$, electric current density $\boldsymbol{J}$, viscous stress tensor $\underline{\tau}$, gas pressure $p=e(\gamma-1)$, viscous dissipation $Q_{\text {visc }}$, Joule $\overline{\bar{d}}$ issipation $Q_{\text {Joule }}$ and speed of light $c$, respectively. The expression $\boldsymbol{u} \otimes \boldsymbol{u}$ stands for the tensor (or dyadic) product of $\boldsymbol{u}$ with itself. An ideal gas law (Eq. (7)) is assumed with $\mathcal{R}$ the gas constant and constant value for $\tilde{\mu}$. The ratio of specific heats, $\gamma$, is taken as $5 / 3$.

\subsection{Numerical approach}

The equations are solved using a high order finite difference approach on staggered grids. This way the conservation requirement of the MHD equations are maintained to machine precision throughout the calculations. To calculate the left hand side of the MHD equations above, variables have to be communicated between the various staggered grids. For this a sixthorder method is applied to derive the partial derivatives and a fifth-order method is used for any interpolation required. The high order approach requires special treatment of viscosity and magnetic resistivity to prevent overshooting from the high order fitting at locations of steep gradients. For this, viscosity and magnetic resistivity are both handled using a combined second and fourth-order method with a discontinuous shock capturing mechanism to provide the highest possible spatial resolution for the given numerical resolution. This effectively gives a viscosity and resistivity treatment that is locally dependent on the physical quantities and therefore give space and time varing values of the respective Reynolds numbers. The density and thermal energy change by many orders of magnitude from the lower boundary to the upper boundary, with a substantial part of this change occurring over the transition region. Interpolation in these variables is done using their logarithmic counterparts. This limits the overshooting and at the same time has a smoothing effect. The solution is advanced in time using a third-order predictor-corrector method (a basic description of the code is available in Nordlund \& Galsgaard 1997, and at http://www.astro.ku.dk/ kg). A challenge for this type of experiments is the requirement to resolve various spatial regions, having a large domain and still being able to compute the solution within a reasonable time. Low resolution tests have shown which regions of the domain require high numerical resolution to provide reliable solutions to the MHD equations. To increase resolution only in these regions, stretched grids are applied in one or more directions. Two regions, namely around the initial location of the flux tube and across the transition region, are crucial. The region around the initial location of the flux tube and across the transition region are crucial. If these regions are not sufficiently resolved, spurious effects, such as unphysical changes in the entropy, occur and the evolution of the emergence process is altered. Below and above these regions less resolution is required as the length scale of the flux tube is either of no importance for the emergence process or have expanded significantly in size. The grid in the vertical direction is, therefore, composed of a central region with an 


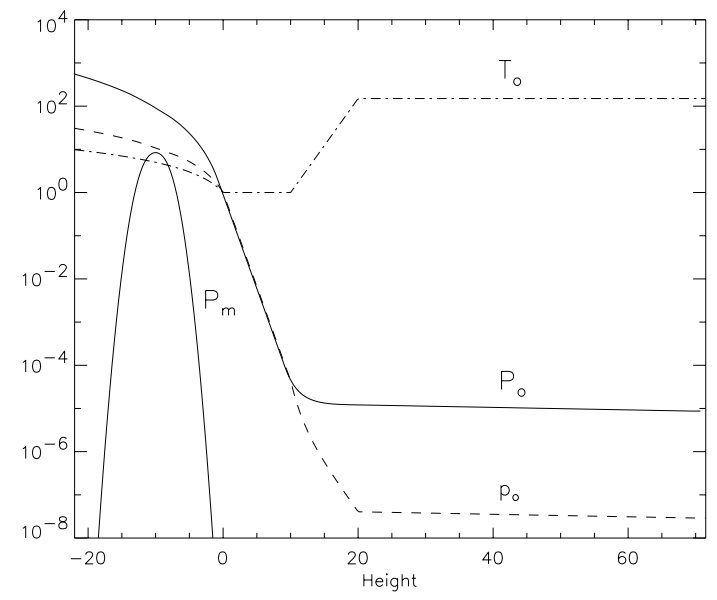

Fig. 1. Distribution of gas pressure (solid), density (dashed), temperature (dash-dotted), and magnetic pressure (solid curve labelled $p_{\mathrm{m}}$ ).

uniform grid covering the initial tube location and the transition region. Outside this, the grid spacing increases using a third order expansion. This has been used to ensure the expansion function has a continuous second order derivative at the transition points. In all experiments in this paper a uniform grid in the horizontal direction is used.

Simple boundary conditions are used: the grid is taken to be periodic in the horizontal directions and closed in the height. The periodic condition has the disadvantage that horizontal information propagates around the domain and returns back after a crossing time and provides a perturbation of the emergence process. The closed boundaries in height prevent information and material from leaving/entering the numerical domain. This implies that the upward propagating waves initiated by the emergence process are bounced back at these boundaries and shortly afterwards interact with the emerging flux tube. To limit the reflection from the closed boundaries, a damping zone has been included at both ends of the domain. Here a fraction of the kinetic energy in the zone is extracted per time unit, with the time scale depending on the location along the damping zone. This works effectively for waves, but has a more limited effect on large shocks.

\section{Background stratification and magnetic tube at time $t=0$}

\subsection{Background stratification}

The initial stratification is a highly simplified model of the actual solar case, including the upper layers of the solar interior, the low atmosphere, transition region and corona. Following the tradition in flux emergence calculations (e.g., Shibata et al. 1989; Nozawa et al. 1992; Matsumoto et al. 1993; Krall et al. 1998; Fan 2001; Magara \& Longcope 2003), for the atmosphere we take two isothermal ranges (which, for brevity, we call the low atmosphere and the corona, respectively) at temperatures $T_{\mathrm{ph}}$ and $T_{\mathrm{c}}$, with $T_{\mathrm{c}}=150 T_{\mathrm{ph}}$. The transition between those layers is made via a region with a steep temperature gradient that we call the transition region, or $T R$ for short. The bottom of the low atmosphere will be called the photosphere and is placed at $z=0$. The values of pressure, density and pressure scale-height at $z=0$, symbolized $p_{\mathrm{ph}}, \rho_{\mathrm{ph}}$ and $H_{\mathrm{ph}}$, will be taken as physical units for the calculation in the rest of the paper. The isothermal corona extends from $z=$ $z_{\mathrm{c}}=20$ to the upper boundary. For the transition region we choose a power-law temperature profile of the form

$T(z)=T_{\mathrm{ph}}\left(\frac{T_{\mathrm{c}}}{T_{\mathrm{ph}}}\right)^{\frac{z-z_{\mathrm{tr}}}{z_{\mathrm{c}}-z_{\mathrm{tr}}}}$

extending between $z=z_{\mathrm{tr}}=10$ and the base of the corona, $z_{\mathrm{c}}$. Underneath the photosphere an isentropic domain with temperature growing linearly with depth is chosen, simulating the average stratification of a convection zone with an adiabatic temperature gradient. Hydrostatic equilibrium is assumed everywhere (Fig. 1).

For the conversion to variables with dimensions, the following values for the units can be used: $p_{\mathrm{ph}}=1.4 \times 10^{5} \mathrm{erg} \mathrm{cm}^{-3}$; $\rho_{\mathrm{ph}}=3 \times 10^{-7} \mathrm{~g} \mathrm{~cm}^{-3} ; T_{\mathrm{ph}}=5.6 \times 10^{3} \mathrm{~K} ; H_{\mathrm{ph}}=170 \mathrm{~km}$. From these values, one obtains a velocity unit, $V \equiv\left(p_{\mathrm{ph}} / \rho_{\mathrm{ph}}\right)^{1 / 2}=$ $6.8 \mathrm{~km} \mathrm{~s}^{-1}$, a time unit $t_{\mathrm{ph}}=25 \mathrm{~s}$ and a magnetic field unit $B_{\mathrm{ph}}=$ $1.3 \times 10^{3}$ Gauss, chosen so that the Alfvén speed is unity for $B_{\mathrm{ph}}$ and $\rho_{\mathrm{ph}}$, and the plasma $\beta$ is 2 for a pressure $p_{\mathrm{ph}}$. Surface gravity is then $2.7 \times 10^{4} \mathrm{~cm} \mathrm{~s}^{-2}$, i.e., the solar value, and $\tilde{\mu}=1$ (in cg units), which is intermediate between the actual solar values in the photosphere and the corona.

The grid for the calculation reported in this paper encompasses the following dimensionless domain in $x, y, z:(-60,60) \times(-70,70) \times(-20,82)$. Resolution is critical in the vertical direction, since the scale height is quite small in the low atmosphere. The stretched grid provides a resolution of $\delta z=0.3$ in all of the convection zone up to the base of the corona. In $x$ and $y$ we use an unstretched grid. For these experiments we typically used $148 \times 160 \times 218$ gridpoints

\subsection{Initial condition for the magnetic tube}

At time $t=0$ a magnetic flux tube is located at a height $z=$ -12 (i.e., some $2000 \mathrm{~km}$ underneath the photosphere) with its axis along the $y$-direction and centered in the $x$ direction. At that depth, the pressure, density and temperature are $80 p_{\mathrm{ph}}$, density $14 \rho_{\mathrm{ph}}$ and temperature $6 T_{\mathrm{ph}}$, respectively. A simple Gaussian profile is chosen for the longitudinal field $B_{y}=$ $B_{0} \exp \left(-r^{2} / R^{2}\right)$, with $r=\sqrt{x^{2}+(z+12)^{2}}$ the radial distance to the axis of the tube, $B_{0}=11.8$ and radius $R=2.5$. The total $(r / R \rightarrow \infty)$ dimensionless flux threading a cross section of the tube is then $2.3 \times 10^{2}$. Using the units of Sect. 3.1, we obtain $9 \times 10^{19} \mathrm{mx}$, as in a small active region (or a large ephemeral active region).

The ratio between the unperturbed stratified gas pressure and the magnetic pressure at the position of the tube axis is $\beta_{0}=$ 14.6. This is substantially lower than the values expected on the basis of the calculations of magnetic tubes rising across the convection zone (Caligari et al. 1995; Moreno-Insertis 2004). However, the timescale of the simulation in its initial stages is proportional to $\beta_{0}^{1 / 2}$ and this sets a severe upper limit to the value we can choose to keep the duration of the simulation 
within reasonable bounds. The value chosen here is a compromise in that at least it yields central values of $\beta$ above unity while the tube is below the photosphere. On the other hand, following Moreno-Insertis \& Emonet (1996), the field lines must be twisted around the central axis to a sufficient degree for the rising tube to retain most of the initial magnetic flux and prevent its being dragged to the wake. The precise criterion given by Emonet \& Moreno-Insertis (1998), valid for high- $\beta$ magnetic tubes with small radius, $R \ll H_{\mathrm{p}}$, (with $H_{\mathrm{p}}$ the local pressure scale-height) is that the following condition be fulfilled:

$\left(\frac{B_{\varphi}}{|\boldsymbol{B}|}\right)_{\max } \gtrsim\left(\frac{R}{H_{\mathrm{p}}}\right)^{1 / 2}\left(\left|\frac{\Delta \rho}{\rho}\right|_{0} \frac{\beta_{0}}{2}\right)^{1 / 2}$,

where $\left(B_{\varphi} /|\boldsymbol{B}|\right)_{\max }$ is to be calculated at the position of the $\max$ imum of the transverse field, $\Delta \rho$ is the density deficit in the tube compared with the original stratification and the right hand side must be calculated at the centre of the tube. It is difficult to fulfill this criterion for the conditions of the present paper, specially since the ratio $R / H_{\mathrm{p}}$ quickly grows along the rise. As in previous flux emergence calculations (e.g., Fan 2001; Magara \& Longcope 2001), we choose helical field lines highly twisted around the central axis (albeit with constant pitch): $B_{\varphi}=\alpha r B_{y}$. Our $\alpha$ is 0.4 . This configuration yields marginal stability as regards criterion (9) for the initial magnetic tube. The field distribution above is not force-free: there is no compelling argument for the vanishing of the Lorentz force, $\boldsymbol{F}_{\mathrm{L}}$, since $\beta$ is well above unity. Yet, the Lorentz force associated with the chosen field distribution is curl-free. Hence, a gas pressure function can be chosen so that the sum of the isotropic gas pressure tensor and the Maxwell stresses is continuous and equal to the gas pressure tensor of the unperturbed stratification. This is a simple choice for the gas pressure at time $t=0$ that also prevents the instantaneous formation of a shock right from the outset.

The choice of density (or, equivalently, entropy) distribution along the tube can importantly influence the outcome of the simulation. Three canonical choices are available: equal density (as used by, e.g., Spruit \& van Ballegooijen 1982; Moreno-Insertis 1986; Caligari et al. 1995); equal entropy (as used, e.g., by Moreno-Insertis \& Emonet 1996; Dorch et al. 1999 ) or equal temperature (as in the classical paper of Parker 1975 and in those of D'Silva \& Choudhuri 1993; and Fan et al. 1994). For flux tubes starting in the deep convection zone, Caligari et al. (1998) have studied in detail the physical basis and consequences of the equal-temperature and equal-density initial conditions. In the present case, for the initial tube to yield a rising $\Omega$-loop, the central part (i.e., the region around $y=0$ ) must be buoyant, or, at least, substantially more buoyant than the feet. Alternatively, the central part could be imparted with an initial upward velocity relative to the feet. Fan (2001) chose a non-uniform distribution of entropy along the tube so that the flanks have equal density, the centre equal temperature as the original unperturbed stratification. This results in the central part being underdense by a relative factor $1 / \beta$, which causes the rapid development of an $\Omega$-loop shape and yields fast emergence at the photosphere. Yet, this choice yields large entropy differences between the centre and the flanks of the tube: in fact the differences may be as large as those associated with the entropy loss through radiation of plasma elements at the top of granules. Such large entropy fluctuations are not expected in supergranules, or, even less, in the deep convection zone (see, e.g., Spruit 1977). Hence, the origin of such fluctuations is difficult to justify in flux tubes that erupt as active regions (ephemeral or otherwise), given their length-scales and assumed origin.

On the other hand, Matsumoto et al. (1993) and Magara \& Longcope $(2001,2003)$ choose an initial tube which has the same density everywhere as the unperturbed stratified atmosphere at the same height. To start the dynamical evolution, the latter authors then pull the central region of the tube upward by artificially adding vertical momentum during an interval (the first $3 \mathrm{~min}$, using their units) at the beginning of the simulation. The added vertical momentum is given following a cosine distribution along the flux tube; the process is stopped when the crest has been imparted a speed of $2.7 \mathrm{~km} \mathrm{~s}^{-1}$. An isodensity initial condition is well justified at the bottom of the convection zone (Caligari et al. 1998). Among other things, the corresponding entropy distribution is uniform along the tube which is more easily compatible with the formation of the tube in a single environment. However, at the shallow initial depth of the present kind of 3D simulations with $\beta$ not far above one, the single value of entropy in the tube is below any value that can be found in the whole convection zone. So, here, again it is not immediate to justify this initial condition on physical grounds. The initial conditions discussed in the foregoing must then be seen mostly as convenient devices to yield a fast time evolution of the tube.

For simplicity, and to facilitate a better comparison with previous literature, in the present paper we adopt one of the initial conditions just explained. In the following sections we, therefore, use an initial density distribution resembling the choice of Fan (2001), i.e., $\rho(x, y, z)=$ $\left[p(x, y, z) / p_{\text {st }}\right] \rho_{\text {st }}(z) \exp \left(-y^{2} / \lambda^{2}\right)$, with $p_{\text {st }}$ the pressure of the unperturbed stratification and $\lambda=20$. Experimenting with other possible choices of initial condition is left for later work.

\section{The emergence of magnetic field: Rise to the photosphere}

\subsection{Initial phase}

The rise through the adiabatically stratified convection zone is driven by buoyancy, with the advance of the tube being countered basically by the drag exerted by the surrounding medium: the Lorentz force associated with the bending of the tube axis is too small at this phase to match the other forces at play. A two-dimensional cut in the $x-z$ plane (i.e., perpendicular to the tube) through the crest of the tube shows similarities with the standard 2.5D cases calculated by Moreno-Insertis \& Emonet (1996) and Emonet \& Moreno-Insertis (1998). There is only limited stretching of the tube in the direction of the axis during most of the rise up to the photosphere. Therefore, using the condition of magnetic flux conservation, we expect the axial field strength to diminish following the law $B_{y}(z)=$ $B_{y}\left(z_{0}\right) \rho(z) / \rho\left(z_{0}\right)$. This law is indeed obeyed by the actual magnetic tube in the present simulations (compare, in Fig. 2, the maximum of the solid-line profiles with the dashed curve): the 


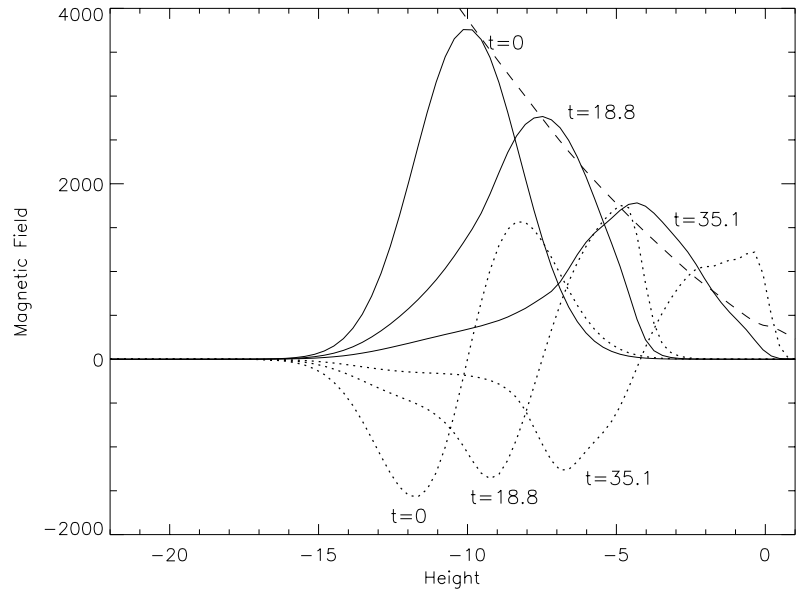

Fig. 2. The axial $\left(B_{y}\right.$, solid lines) and transverse $\left(B_{x}\right.$, dotted lines) field components as a function of height along the vertical axis at the centre of the box $(x=y=0)$ at three different times during the rise to the photosphere. The initial Gaussian profile (solid line at $t=0$ ) decreases as the tube rises toward the surface, following the simple expansion law $B_{y} \propto \rho$ indicated by the dashed line. The transverse component decreases more slowly than the axial field, so that the field line pitch increases as the tube expands.

slight deviation, especially in the final time shown, comes from the stretching of the tube in the axial direction along the rise. On the other hand, the stronger buoyancy of the tube centre makes it advance with respect to the periphery above it, so that the azimuthal field component becomes enhanced at the front and weakened at the rear in the initial stages. This is clearly visible in Fig. 2 by comparing the dotted lines at time $t=0$ and $t=18.8$. Toward the end of the rise up to the photosphere, however, all components of the field decrease due to the continued expansion of the tube. The decrease of the azimuthal components, however, occurs at a slower rate than for the axial component, as expected from flux conservation considerations. As a result, the field line pitch increases as the tube rises. Concerning the flows around the tube, the trailing wake does not reach the degree of development apparent in the cases studied by Emonet \& Moreno-Insertis (1998), possibly because of the higher Reynolds number attainable in the $2.5 \mathrm{D}$ experiments. Full agreeement was found in that the 3D experiments with a high level of twist (as those presented in the current paper) retain the large majority of the initial magnetic flux as a single tube rising in compact form, whereas test runs carried with a lower twist yield the formation of a broad wake with two vortex rolls containing a substantial fraction of the initial magnetic flux of the tube.

\subsection{Initial upgoing shock}

The start of the rise of the central section of the tube causes a compression wave to be launched upward. The wave has small amplitude while in the convection zone. The steep density gradient in the low atmosphere makes it grow in amplitude and steepen to form a shock wave, reaching a large amplitude upon rising several scale heights above the photosphere (Fig. 3). In the low atmosphere, which has an isothermal stratification

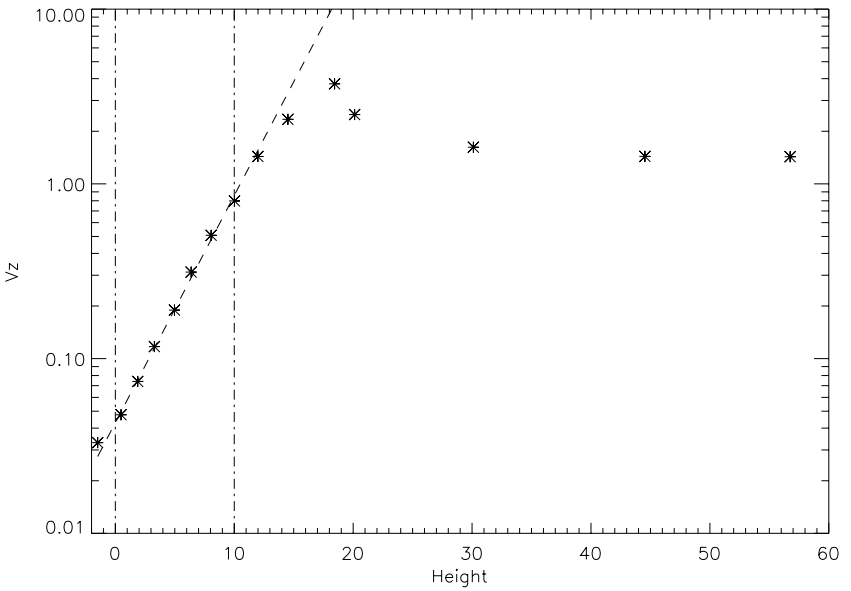

Fig. 3. The post-shock speed as a function of time follows an exponential growth as the shock moves between the base of the photosphere at $z=0$ and the lower level of the transition region $(z=10)$. In the transition region the growth is slower due to the steep temperature rise. In the corona, finally, the large scaleheight leads to a very slow variation of the shock strength with height.

between $z=0$ and $z=10$, the post-shock speed closely follows an exponential law, as expected from the exponential decline of the background density with height. A clear deviation from the exponential behavior occurs in the transition region (as seen in the figure for $z>10$ ). There, the unperturbed temperature (hence the sound speed) steeply increases with height and this leads to a slower increase, first, and, then, to a decrease of the post-shock speed. When the shock finally enters the corona, the shock parameters change with height very slowly. This is a consequence of the high coronal temperatures which yield a background scaleheight of $100 H_{\mathrm{p}}$.

The simulation shows further agreement with the theory of shock wave propagation in atmospheres: in an isothermal stratification, an upgoing compression pulse is followed by a wake in which the plasma oscillates vertically with a frequency asymptotically approaching the acoustic cut-off frequency, $v_{\mathrm{c}}=c /(2 H)$, (Lamb 1932; Kalkofen et al. 1994). The shock in the present simulation develops that structure: in the low atmosphere a vertical oscillation appears behind the upgoing shock. The first change of sign of the oscillation occurs when the original shock is already propagating at coronal heights. The upward-directed speed in every half-period of the oscillation causes the upward launch of a new shock wave that ends up propagating through the higher levels of the atmosphere. The evolution in the low atmosphere (and even in the first several scale heights of the transition region) is strongly reminiscent of the nonlinear results of Kalkofen et al. (1994).

\subsection{Arrival at the photosphere}

The main body of the rising tube acquires a rise speed of about 0.25 (some $1.7 \mathrm{~km} \mathrm{~s}^{-1}$ ) while still in the convection zone, so that it takes up to time $t \equiv t_{\text {surf }}=30(12.5 \mathrm{~min})$ for its crest to reach the photosphere. By that time, the centre of the tube is still at a depth of some $850 \mathrm{~km}(z=-5)$. The density contrast between initial depth and photosphere (factor 14) 
causes a marked expansion of the tube. In fact, vertical and horizontal expansion rates are different, since the former has to do extra work against gravity: indeed, we see a large horizontal expansion with only a moderate expansion in height (a result anticipated by Spruit et al. 1987; and also visible in the figures of some of the $2.5 \mathrm{D}$ simulations cited in the introduction). To quantify this effect, we consider a vertical cross section of the tube, measure the full width at half maximum of the magnetic field both in the vertical direction upward of the tube centre and horizontally from it and calculate the ratio of those two quantities: the result at $t=t_{\text {surf }}$ is 3 . When the periphery of the tube reaches the surface (see, e.g., the solid and dotted curves at $t=35$ in Fig. 2), the field lines are almost at an angle of $90 \mathrm{deg}$ to the initial direction of the axis, following the pitchangle increase described in Sect. 4.1 The plasma beta of the mass elements reaching the photosphere is 4 , not far from the actual solar values: recent observational results obtained with Stokes polarimetry by Martinez-Pillet (private communication) yield values of $600 \mathrm{G}$ for the rising magnetic field close to the photosphere, a figure which compares favorably with the values obtained in the present simulation.

An isothermal stratification (as chosen for the low atmosphere, $0 \leq z \leq 10$ ) has logarithmic temperature gradient $\nabla=$ -0.4 , and is, therefore, stable against the convective instability. The further upward motion of the tube thus involves lifting overdense plasma against gravity. The tube cannot continue its rise unimpeded, and reduces its velocity by about a factor 2 . This, in turn, produces a relative pressure hill at the point of emergence: the upward moving plasma elements at lower levels get deflected sideways (except those strictly on the vertical midplane of the tube) and the area covered by magnetic plasma at the photosphere increases with time. Thus, the tube enters an intermediate phase, characterized by a slower rise of the magnetized plasma upward of the photosphere and the enhanced expansion in the transverse direction.

The further evolution occurs on the basis of the buoyancy instability experienced by the plasma above the photosphere. Buoyant instabilities, both in magnetized and unmagnetized plasmas, have been thoroughly studied in the literature (Newcomb 1961; Yu 1965; Acheson 1979; see also the review by Hughes \& Proctor 1988). The fundamental mechanism is the fact that a magnetic intensity distribution which decreases with height, by reducing the gas pressure gradient, promotes the top-heavy instability, i.e., as when heavier fluid overlies lighter one. In the present case, however, the strongly stabilizing effect of the subadiabatic stratification above $z=0$ can effectively thwart the development of the instability: any plasma packet rising adiabatically would soon become heavier than the environment. The precise criterion for instability against perturbations which bend the field lines reads (Newcomb 1961; Thomas \& Nye 1975; Acheson 1979)

$-H_{\mathrm{p}} \frac{\partial}{\partial z}(\log B)>-\frac{\gamma}{2} \beta \delta+{\tilde{k_{\|}}}^{2}\left(1+\frac{{\tilde{k_{\perp}}}^{2}}{{\tilde{k_{z}}}^{2}}\right)$,

with $\delta$ the superadiabatic excess, $\delta=\nabla-\nabla_{\text {ad }}, \nabla$ the logarithmic temperature gradient and $\nabla_{\mathrm{ad}}$ its adiabatic value, and $\tilde{k_{\|}}, \tilde{k_{\perp}}$ the wavenumbers of the perturbation in the two horizontal directions parallel and perpendicular, respectively, to the magnetic field in units of the local scaleheight (Eq. (10) corresponds to the expression given by Acheson 1979, rewritten with our variables). The left-hand side term just describes the instability-promoting effect of the decrease of $B$ with height in the upper part of the tube (as apparent in Fig. 2). A crucial term, containing the effect of the stratification, is the $\beta \delta$ term on the right-hand side of the inequality. For an isothermal stratification $\delta=-0.4$, which causes strong stabilization. However, this term is multiplied by $\beta$ and, therefore, becomes small as the magnetic pressure grows above the local gas pressure when the tube crosses the photosphere. In the actual experiment, when the upper layers of the rising tube reach the photosphere, the left-hand side term is well below the stratification term on the right. As the topmost magnetic layers cross the photosphere, their ratio quickly changes as the plasma $\beta$ decreases. Finally, the ratio goes below 1: precisely at this time ( $t \sim 45$, i.e., some $10 \mathrm{~min}$ after arrival at the photosphere), the subadiabatically stratified plasma above it can no longer check the further rise of the tube and the buoyancy instability is launched, which takes the magnetized plasma all the way up to the corona. By then the uppermost layers of the tube are at $z=6$ and its centre has reached $z=-2$. We think this also explains (at least partially) why the duration of the intermediate phase is proportional to the intensity of the field reaching the photosphere, hence also to the field strength of the tube at time $t=0$ (a fact already pointed out by Magara 2001): the weaker the field that reaches the photosphere, the longer it takes for the plasma $\beta$ to go down sufficiently so that the right-hand side of inequality (10) becomes low enough. The instability described here is related with, but is different from, the simple magnetic Rayleigh-Taylor instability of a heavy fluid overlying a lighter, more strongly magnetized one, with a sharp interface separating them. The gradients of field and entropy, in particular, are absent from the simple RT-instability theory, but they are crucial to determine threshold and growth rate. To our knowledge, criterion (10) has not been used in the previous literature cited in the introduction.

\section{The expansion into the higher atmosphere}

\subsection{Runaway expansion of the magnetized plasma and downflows in the periphery}

For the parameters chosen in the simulation described here, the expansion into the higher levels of the atmosphere (starting around $t=60$ ) occurs in a runaway fashion. A clear sign of this is that the distribution of magnetic pressure with height above the photosphere in the expanding magnetized plasma volume is a few orders of magnitude larger than the gas pressure. The vertical velocity of the rising plasma quickly increases to $v_{z} \sim 1$ when the front of the expanding volume reaches the bottom of the transition region (at $z=10$ ). By time $t=70.4$ the velocity profile along the central vertical axis $(x=0, y=0)$ within the upgoing magnetic volume shows a monotonic increase of the vertical velocity with height between $v_{z} \sim 0.1$ at $z=2$ and $v_{z} \sim 2.5$ at $z=27$, where the front of the tube is located (Fig. 4, upper panel). 

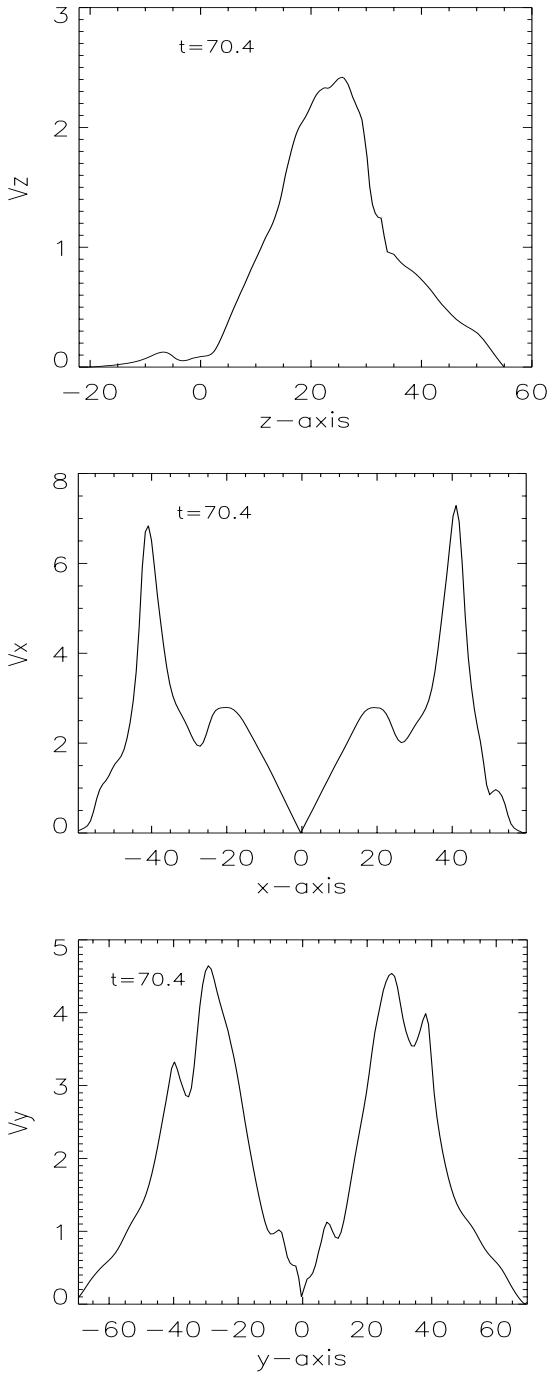

Fig. 4. Anisotropic expansion of the rising plasma. The diagram on the top shows the vertical velocity profile along the $z$ axis going through the centre of the box. A typical expansion profile between the photosphere and the lower levels of the corona is apparent, with maximum velocity $v_{z}(\max ) \approx 2.5$. The diagrams in the center and on the bottom show the distribution of horizontal speed in the plane $z=20$ along the $x$ and $y$ axis, respectively. A clear expansion profile is found here as well, but the velocities are much higher: $\left|v_{x}(\max )\right| \approx 7$ and $\left|v_{y}(\max )\right| \approx 5$.

The runaway expansion also takes place in the horizontal directions, in fact with higher speeds than in the vertical direction. For instance, at $t=70.5$, the $x$-component of the velocity along the horizontal plane $z=20$ (base of the corona) shows a quasi-monotonic expansion profile away from the centre, reaching a speed of $\left|v_{x}\right| \sim 7$ at $|x| \sim 40$, which is where the boundary of the magnetized region is located (see Fig. 4, central panel). In a similar manner, the $y$-component of the velocity along the same horizontal plane shows that the tube expands sideways with a maximum velocity of $\left|v_{y}\right| \sim 5$ at $|y| \sim 30$ (Fig. 4, bottom panel).

The resulting 3D image of the magnetic region can be visualized using isointensity surfaces of the magnetic field (Fig. 5, upper panel) or the corresponding isointensity contours within
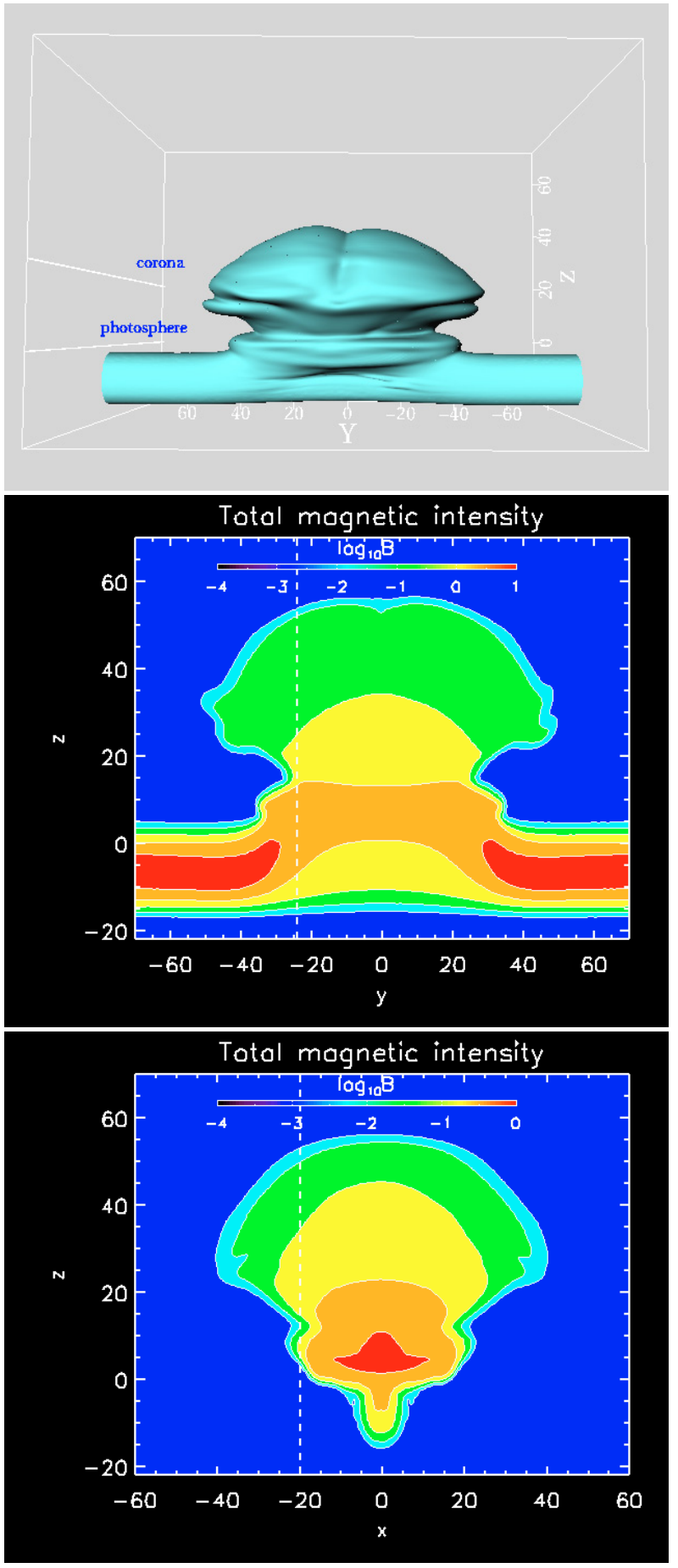

Fig. 5. 3d (upper panel) and $2 \mathrm{~d}$ (middle and bottom panels) visualization of the magnetic field strength of the expanding tube at $t=70.46$. In the upper panel, the isosurface corresponds to $|\boldsymbol{B}|=10^{-3}$ (maximum $|\boldsymbol{B}|$ at this time is 2.4). The color map shows the isointensity contours of the expanding tube in the midplane along the $y$-direction (middle panel) and $z$-direction (bottom panel). The dashed lines are auxiliary lines for Fig. 6.

the vertical midplane along the $y$-direction (Fig. 5, central panel) and along the transverse $(x)$ direction (bottom pannel). The magnetized volume has the shape of a narrow region at the original level in the convection zone around $z=-10$, suffering a first expansion up to the photosphere and then a second 

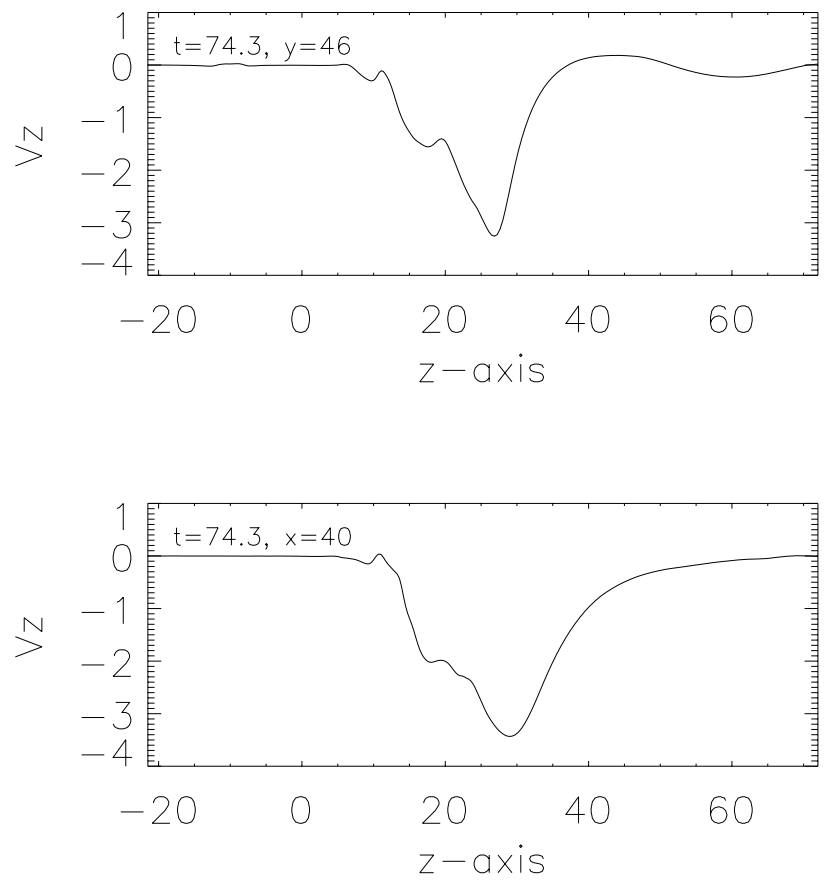

Fig. 6. Downflows in the periphery of the rising magnetic region as the tube enters the corona $(t=74)$. Shown is the distribution of $v_{z}$ with height along the following lines: (upper panel) vertical line situated within the $x=0$ midplane of the box at $y=46$ (shown as a dashed line in Fig. 5, central panel); (lower panel) vertical line situated within the $y=0$ midplane of the box the tube at $x=40$ (shown as a dashed line in Fig. 5, bottom panel). The maximum downflow speeds in the figure correspond to about $24 \mathrm{~km} \mathrm{~s}^{-1}$.

(and much more marked) expansion in the atmosphere, as a cake being baked which is contained in its mould in the lower levels but overflowing it and expanding in all directions above it.

The plasma surrounding the rising magnetized ball also shows some dramatic dynamics: it gets pushed sideways by the expanding plasma and reaches similar horizontal velocities. Also, because of mass conservation, the fast rise and expansion of the magnetic plasma causes strong downflows along its periphery (see Fig. 6). Typical values for the downflow velocity can be as high as $\left|v_{z}\right| \sim 2$ (i.e., $\sim 14 \mathrm{~km} \mathrm{~s}^{-1}$ ) at the height of the transition region and $\left|v_{z}\right| \sim 3.5\left(\sim 24 \mathrm{~km} \mathrm{~s}^{-1}\right)$ above the base of the corona. These values compare favorably with velocities observed along the line of sight in developed active region loops at chromospheric and coronal levels (Brekke et al. 1997; Chae et al. 1998).

\subsection{The magnetic field lines}

Additionally to isosurfaces, actual field lines are necessary to illustrate the structure and topology of the magnetic field. Previous authors (Fan 2001; Magara \& Longcope 2001, 2003) have shown the shape of three individual field lines (the tube axis and two more peripheral lines), pursuing them along time. In this section we would like to show whole sets of field lines at a time when the eruption into the corona is well advanced, to better visualize the global geometry of the magnetic field and the linkage between different parts of the volume.
Figure 7 shows three sets of field lines (in blue, green and red, respectively) all of them calculated at time $t=72$. Three views are shown: front (uppermost panels), side (middle panels) and top view (lower panels). To facilitate the visualization, an isosurface of the total magnetic field strength at $|\boldsymbol{B}|=0.2$ is added (maximum magnetic field strength at that time is $|\boldsymbol{B}|=2.18$, located in the legs of the tube). The first set of field lines (solid lines in blue color, left side) is drawn starting from points close both to the tube axis and to the $y$-boundary on the right side of the computational domain. These lines remain inside the volume limited by the isosurface in the flanks of the tube. Only their windings in the central part traverse the isosurface (and are thus visible in the figure). There, they reach moderate heights, at any rate below the corona.

The second set (green solid lines, left side) consists of field lines starting right at the isosurface close to the $y$-boundary. They remain by the isosurface in the flanks but, upon reaching the central section, they describe large loops linking the photosphere with the upper levels of the low atmosphere. The sideways expansion in the axial direction experienced by the plasma along the rise is clearly visible here (see the front view in the top-left panel). The succesive windings as one goes from the vertical $x-z$ midplane toward the flanks are increasingly inclined, with each individual winding being contained in a curved surface. These fieldines have an orientation more perpendicular to the central axis of the tube than the innermost (blue) fieldlines around the central part (see the top view shown in the bottom-left panel). This shape is visible already in one of the field lines shown by the authors cited above.

The third set (right side of Fig. 7, colored in red), consists of field lines starting from points in the flanks of the tube slightly outside the volume enclosed by the isosurface. This set of field lines reaches the highest levels of the magnetized plasma, located well above the original interface with the corona. Their shape illustrates, more clearly than the second set, the strongly azimuthal nature of the field lines in the central region and the effects of the predominance of the lateral expansion of the upcoming plasma.

The isosurface shown in the figure, in turn, is particularly interesting since it clearly shows (a) the flattened structure of the magnetic region at the photosphere, following the processes discussed in Sect. 4.3, and (b) an S-shaped structure along the $y$-direction (clearly discernible in the top view panels). The $\mathrm{S}$-shape is a consequence of the gradual conversion of field line twist into writhe along the calculation. S-shapes have also been found and discussed by previous authors (e.g., Matsumoto et al. 1998; Fan 2001; Magara 2001).

\section{3. adiabatic cooling and resulting stratification}

Further features associated with the runaway expansion are the adiabatic cooling and the deviation of the stratification from the initial hydrostatic profile. The present simulation has no radiative transfer or cooling built in and so the moving plasma must behave adiabatically wherever the gradients are not so large that the diffusion terms become important. On the other hand, 

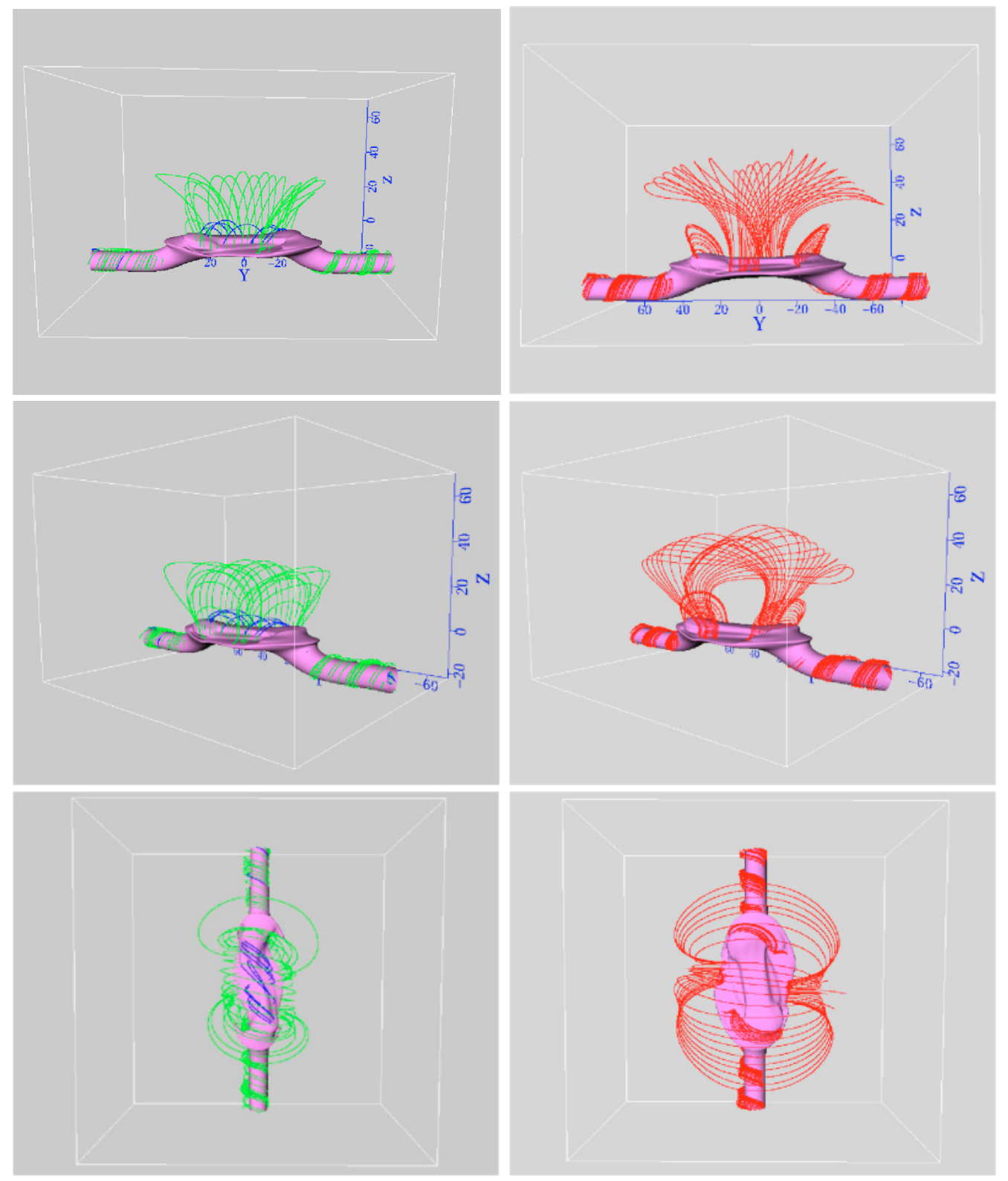

Fig. 7. Eight panels showing different views of the fieldlines at time $t=72$, when the rising magnetic plasma has already reached coronal heights. The panels on the left side of the figure show the fieldlines closer to the main axis of the tube (blue) and those who reach the top layer of the transition region (green). The panels on the right side (in red color) show more clearly the runaway expansion of the fieldlines into the corona.

the initial stratification has two isothermal ranges, which have a steep increase in entropy. Hence, the expanding plasma of the magnetic tube must show marked differences in its pressure, density and thermal distributions with height as compared with the pre-existing distributions at time $t=0, p_{\mathrm{st}}, \rho_{\mathrm{st}}, T_{\mathrm{st}}$. In fact, the profiles at time $t=78.7$ show (1) much lower gas pressure and temperature than the original stratification, with a maximum ratio of a few orders of magnitude; (2) dominating magnetic pressure, well above the original gas pressure, and with the plasma $\beta$ going down to $O\left(10^{-3}\right)$ and $(3) \rho(z) / \rho_{\text {st }}(z)$ of order one in the lower atmosphere but well above that value closer to the front of the expanding tube, in fact, with a maximum of 100. It is remarkable that the rising plasma ball can push up such dense matter, compared with the density of the preexisting atmosphere. The reason is the high value of the magnetic pressure gradient (as apparent in the left panel of Fig. 8), which is well above the (gas) pressure gradient in the background hydrostatic stratification.

The thermal behavior of an actual active region in the Sun must deviate from the above. In the low atmosphere, radiative transfer in the rising plasma will be the main thermal agent causing its entropy to vary in time, with the timescales for the radiative exchange in some spectral lines being as short as seconds. In the higher levels of the atmosphere, coronal heating, possibly ohmic heating associated with nanoflare reconnection or wave dissipation, will increase the temperature of the magnetic plasma to coronal levels. More complicated models will have to deal with these additional aspects. The current results 

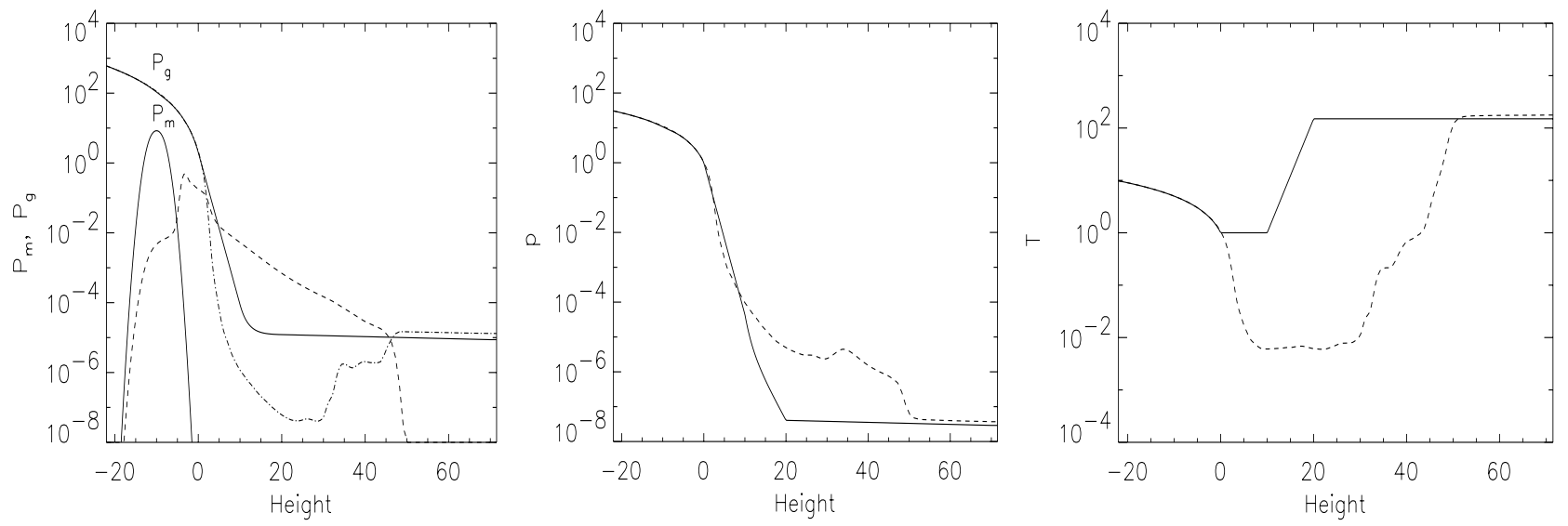

Fig. 8. Height distribution of pressure, density and temperature at time $t=0$ (solid lines) and at time $t=78.7$ (dashed and dash-dotted lines). Left panel: magnetic pressure (solid and dashed lines) and gas pressure (solid and dash-dotted lines). Central panel: density. Right panel: temperature. The emerging plasma is magnetically dominated, with the plasma beta decreasing to order $10^{-3}$ in it, and has a higher density (factor 100) and lower temperature (factor $10^{4}$ ) than the original coronal values.

can be of interest in that they show how cool the emerging plasma can get if the heating mechanisms are not in operation (or are not fast enough).

\section{Synthetic magnetograms and velocity fields at different levels}

The lack of radiative transfer and explicit coronal heating processes in the equations we are using limits the possibilities of carrying out comparisons of our results with observations of the birth and rise into the corona of an active region. However, it may be instructive to construct synthetic magnetograms and velocity vector fields of the active region produced in the current simulation simultaneously at different heights in the box. Figure 9 shows filled contours of the surface distribution of $B_{z}$ on horizontal cuts of the box situated at a depth of $850 \mathrm{~km}$ $(z=-5)$, the photosphere $(z=0)$, the base of the transition region $(z=10)$ and the base of the corona $(z=20)$. The corresponding distribution of the total velocity is shown by arrows which are superimposed on the above magnetograms. For clarity, in the two topmost panels only two cuts, corresponding to the convection zone and the photosphere, are presented; the two lowermost panels, in turn, show cuts for the photosphere, transition region and corona. For the photosphere, magnetograms and velocity fields have been presented by previous authors (Fan 2001; Magara \& Longcope 2001, 2003); our results for that layer agree in broad terms with theirs.

When the top part of the magnetic tube first reaches the photosphere ( $t=30.4$, Fig. 9, top-left, upper slice), the vertical field component, $B_{z}$, yields a simple bipolar structure constituting an incipient active region. Its orientation at this very early stage of evolution is perpendicular to the central axis of the tube (North-South, or N-S, orientation in the following, for short). This is due to the high level of twist of the field lines in the periphery of the tube (see Fig. 2 and Sect. 5.2) together with the fact that the flanks of the magnetic tube are not yet sufficiently inclined for the axial field component to have a large enough projection on the vertical direction. A magnetogram in the convection zone at the same time (lower cut in the same panel), shows two N-S bipolar structures, one at each flank of the magnetic tube, which, again are due to the transverse field component and the small inclination of the tube axis at that level. Also there, we see a strong upflow in the central regions of the slice in the wake of the emerging active region, with a weak downflow close to the footpoints. Strong upflow is also apparent in the photosphere (upper slice) where the velocity distribution has a divergence pattern with horizontal flows around the bipolar region. These flows are directed away from the site of emergence, both in the $\mathrm{N}-\mathrm{S}$ and $\mathrm{E}-\mathrm{W}$ directions.

At a somewhat later time ( $t=42.4$, top-right panel), the emergence process has progressed considerably: in the photosphere, upflow at the centre and horizontal motions in the periphery are more marked than in the panel on the left; the magnetized area in the photosphere is now much larger, the feet of the tube in the convection zone have receded toward the $\mathrm{E}-\mathrm{W}$ boundaries. The inclination of the flanks is now larger, which gives more weight to the positive (negative) $B_{z}$ component on the East (West) side of the magnetogram. As a result, we see in the figure (upper panel) that the N-S bipolarity at the photosphere is beginning to turn into an E-W alignment. There is also a shear flow along the neutral (axis-aligned) line, with the horizontal velocity component pointing toward the East on the north side of the neutral line and toward the West on the south side. As shown by Manchester (2001), this is a consequence of the Lorentz force, which is no longer small in the photosphere, and the frozen-in condition for magnetic field and plasma: the field line twist thus causes an extra pull to the plasma in the direction parallel (antiparallel) to the tube axis on the north (south) side.

At later stages of emergence $(t=57$ and $t=67$, shown in the two lower panels of Fig. 9), the magnetogram in the photosphere (lowermost cut in the panels) shows the magnetic distribution to have turned into a bipolar structure roughly oriented in the $\mathrm{E}-\mathrm{W}$ direction, as one expects from active region observations: the polarity seen on each side corresponds to the sign of the component of the field along the main axis of the tube; its flanks have now a sufficient inclination for that polarity 


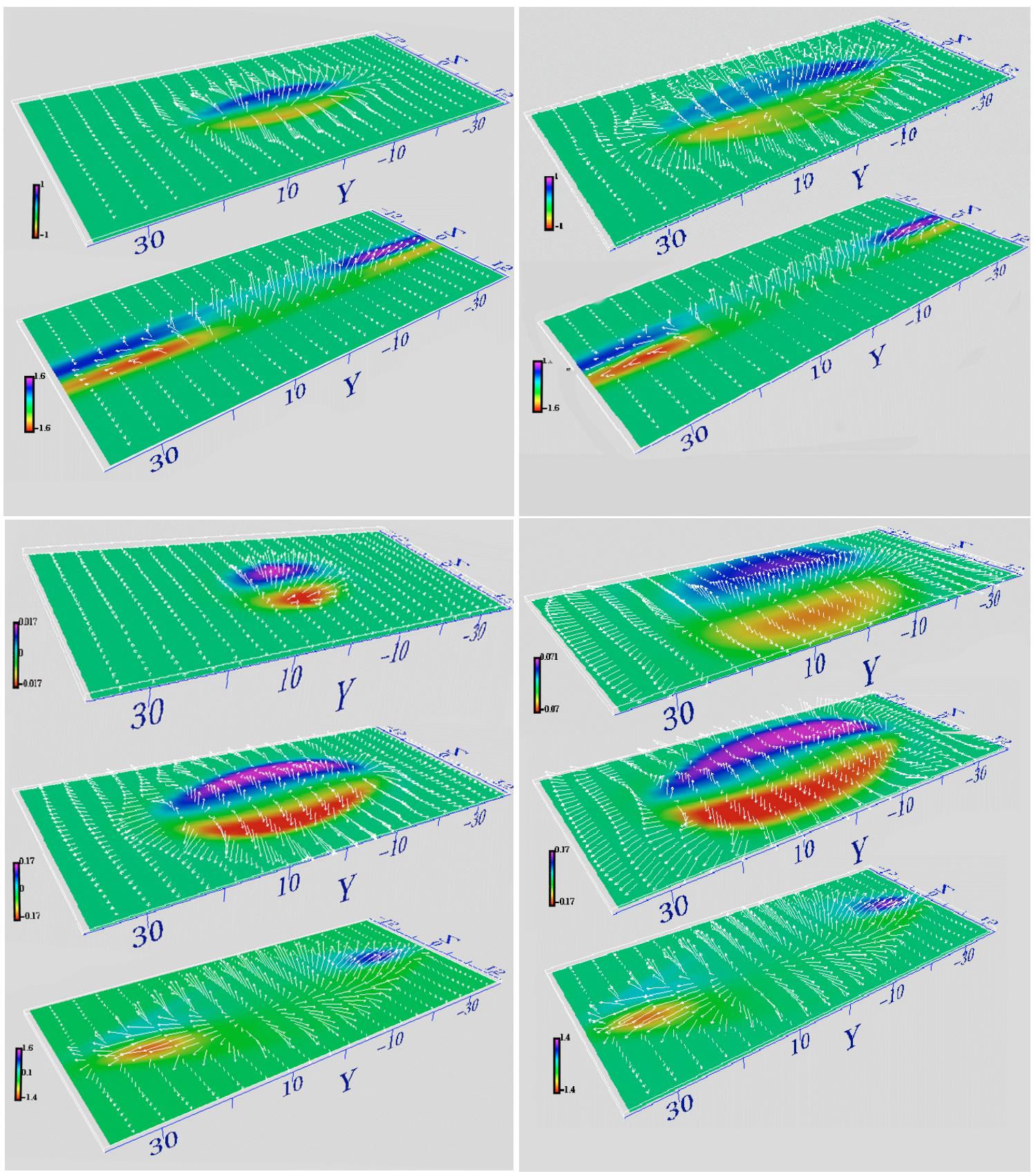

Fig. 9. Synthetic magnetograms and velocity vector distribution on two-dimensional cuts at different times and heights in the convection zone and atmosphere. The panels in the top row show cuts at $z=-5$ (convection zone, lower slice) and $z=0$ (photosphere, upper slice) at time $t=30.4$ (left panel) and $t=42.4$ (right panel). The panels at the bottom correspond to a later stage of the emergence $(t=57$, left, and $t=67$, right) and show the photosphere (lowermost cut), the transition region ( $z=10$, central cut) and the corona ( $z=20$, top cut). The colour-scaled maps correspond to the vertical magnetic field, $B_{z}$; the arrows correspond to the velocity vector.

to be predominant. On one side of the main polarity one sees a patch of weaker, opposite polarity magnetic field. This is still due to the field line twist and is a remnant of the situation described for the top panels in the figure; the opposite polarity patches slowly fade away as the flanks of the tube become more inclined. The panels include a cut at the base of the corona ( $z=20$, topmost cut in each panel) and a cut at the base of the transition region ( $z=10$, central cut). In all of them, the $B_{z}$-distribution shows $\mathrm{N}-\mathrm{S}$ oriented bipolarity. In the panel on the left, the magnetized region has just reached the base of the corona; the velocity field there (slice at the top) shows an upflow coinciding with the site of emergence. In the central cut of the left panel and in both the TR and coronal cuts on the right, in contrast, the velocity arrows indicate a strong horizontal expansion accompanied by a weaker upflow component of the velocity. All of this is in agreement with the magnetic field intensity isosurfaces (Fig. 5) and field line diagrams (Fig. 7) described in Sect. 5.2, which show the predominance of the expansion in the horizontal directions in terms of velocity values 
and stretching rates as compared to the expansion in the vertical direction.

\section{Emergence of magnetic flux into an atmosphere with preexisting horizontal magnetic field: Evidence of reconnection}

In the previous sections the dynamics of a magnetic tube rising into a field-free atmosphere has been discussed. This section investigates the implications of the presence of a simple background coronal magnetic field prior to the emergence of the magnetized region from the convection zone. The initial conditions of Sect. 3.1 are thus altered by including a plane-parallel magnetic field above a certain height, as follows. The background magnetic field is taken to be uniform in the $x y$-plane as well as at all heights above $z=10$. It shows a smooth decrease in strength to zero in the lower part of the transition region. To maintain the hydrostatic balance of the atmosphere, the gas pressure in those heights is decreased accordingly. The background magnetic field is everywhere chosen to point in the negative $x$-direction: $\boldsymbol{B}=\left[B_{x}(z), 0,0\right]$ (S-N direction). The sign and orientation of the coronal magnetic field is such that it optimizes the magnetic reconnection with the magnetic flux system rising from the convection zone: as shown in the previous sections the upper parts of the upcoming magnetized region are predominantly oriented in the $\mathrm{N}-\mathrm{S}$ direction. A precursor to this experiment was done carried out by (Shibata et al. 1992), although only in 2D. As explained in the following, there are important differences between two- and threedimensional experiments.

The evolution of the emerging flux tube, up to the time where the flux of the rising tube comes into contact with the coronal magnetic field, is nearly identical with the zero coronal magnetic field case. The minor differences between the two cases are due to the small change in the characteristic propagation speed in the corona.

As the magnetized plasma rises through the transition region, the orientation of the emerging magnetic field is nearly parallel to the $x$-direction as described earlier. With the particular choice of $\boldsymbol{B}$ made for the background corona and flux tube, when the two systems get in contact their magnetic fields are antiparallel. For the flux tube to emerge into the corona, it has to either push the ambient magnetic field away or reconnect with it to open up a volume into which the emerging field can expand. Figure 10 contains a series of panels showing magnetic field lines traced from the boundaries in the $x$-direction with two different orientations. The starting points for the field line traces are chosen to have a constant height in $z$ and a uniform distribution along $y$. The colours indicate starting points at three different heights, traced from alternative locations near the $x$ boundary. The time series shows how the field lines maintain their clear horizontal direction despite the continual buffeting by waves in the corona. As the upper part of the rising flux tube reaches the lower part of the coronal magnetic field, the coronal magnetic field is pushed upwards and a current concentration is formed that has a dome-like structure (just as seen in Fig. 12). The current magnitude increases with time and magnetic reconnection eventually starts, firstly at the summit point of the emerging flux and later on by spreading down along the sides ( $\mathrm{E}-\mathrm{W}$ direction) of the emerging region in a direction aligned with the flux tube. This creates two new distinct classes of field lines which connect parts of the outer layers of the flux tube to the coronal magnetic field. One such class contains field lines from the front/left $x$-boundary of the domain that connect to the flux tube and reach the left/back $y$ boundary of the image, represented by the light blue field lines. The other class connects the field lines from the right/front $y$ boundary of the flux tube to the back/right $x$-boundary of the box, shown by the blue field lines. Hence, we are witnessing full $3 D$ reconnection. In $2 \mathrm{D}$, the traditional $X$-type reconnection connects magnetic field from above and below the current sheet region and produces newly connected field lines in the outflow region that are formed from half of the field lines in each of the inflow regions (Priest \& Forbes 2000). Here the situation is similar for the local region in the neighbourhood of the reconnection site. The difference from the $2 \mathrm{D}$ description increases with horizontal distance from the reconnection site. This is because the field lines no longer stay in the 2D plane. It is the magnetic field component along the flux tube that provides the preferred direction of connectivity for the field lines on either side of the symmetry line along the emerging flux.

If magnetic reconnection only took place at the summit of the emerging field, then only field lines from a region in the vicinity of this point would undergo magnetic reconnection. For the tube to rise significantly into the corona, it would have to push the rest of the coronal flux sideways, resulting in a significant compression of the magnetic field in the horizontal direction. This is not seen in the experiment. Instead, it is found that the extension in the $y$-direction $(\mathrm{E}-\mathrm{W})$ of the flux connecting from the $x$-boundaries to the flux rope is comparable to the area occupied by the rising flux tube in the $y=0$ plane, leaving only a small compression in the horizontal direction of the coronal magnetic field. This implies that the magnetic field lines from the flux tube reconnect nearly as fast as they are brought into contact with the coronal magnetic field and that reconnection must take place over a large part of the $y$-extent (E-W) of the rising loop at any height in $z$. From Fig. 10 it is apparent that coronal field lines from the same $z$-heights connect onto the same cylindrical radii of the flux tube, despite the fact that they reconnect at different times. This supports a picture whereby the outer layers of the flux tube are slowly peeled off by the reconnection process in a similar manner to removing layers of an onion. Therefore, the larger the $z$-height of a coronal layer that connects to the tube, the smaller the radius of the flux tube that it connects to. As a consequence, this requires the rising flux tube to have a larger amount of flux than the coronal flux of the region that the tube is rising into. If the flux in the rising tube is too small, then it cannot rise into the corona and produce its own magnetic structure. It will instead totally reconnect with the coronal magnetic field, so that the feet of the magnetic tube will end up connected to different ambient sources. In other words, the two polarities of the newly-emerged active regions would be connected to different magnetic areas in the atmosphere instead of to each other.

In $2 \mathrm{D}$, magnetic reconnection can easily be recognized by the presence of a quadrupolar vorticity structure aligned 

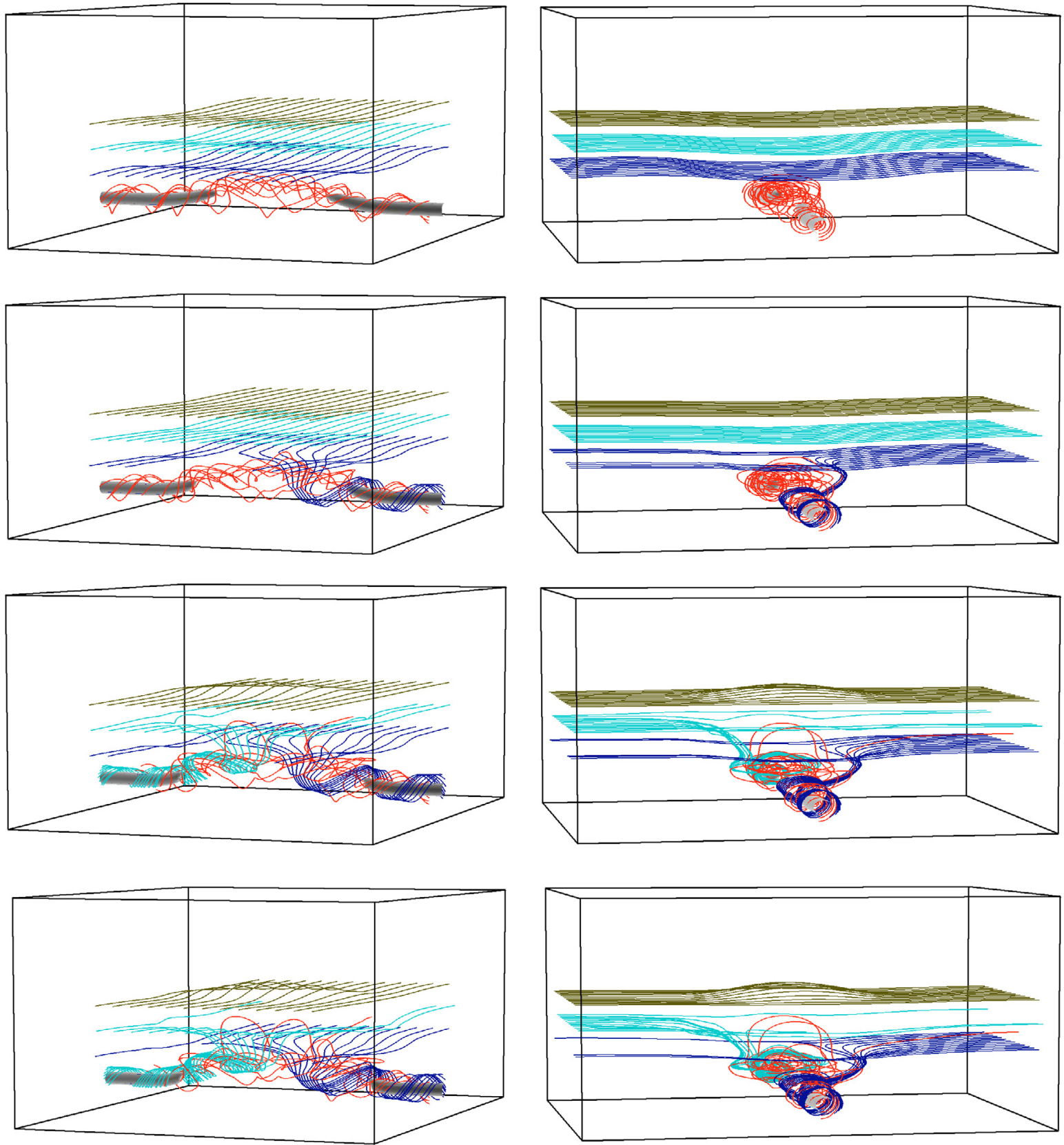

Fig. 10. Magnetic field line traces and isosurfaces of strong magnetic field in time show the changes in the connectivity as the flux tube emerges into the coronal magnetic field. The images represent the times 52.0, 66.7, 80.8, and 85.8 from the top down. Two different orientations of the domain are shown from left to right. The box represents the full size of the domain. From the right column the dark blue and green field lines are traced from the right side boundary, the light blue are traced from the left side boundary and the red field lines are traced from a line going through the isosurface.

with the separator lines, e.g., (Sonnerup 1988). Figure 11 shows the $x z$-plane along the centre of the tube, showing the $y$-component of the vorticity. The top and bottom panels of the figure correspond to the cases without and with background coronal magnetic field, respectively. Only in the case with background magnetic field do we see a clear quadrupolar structure, namely the upper $x$-structure in the figure, the lower one being due to a sudden increase of emergence pushing its way up. The $x$-structured vorticity pattern can be taken as a telltale signature of ongoing reconnection, even if we had no magnetic field line traces to prove it.
The reconnection of the magnetic field lines between the rising flux tube and the overlying coronal field creates plasma heating due to Joule dissipation. In Fig. 12, the impact of Joule dissipation on the increase in thermal energy is illustrated by means of isosurfaces of $Q_{j} / \rho$, with $Q_{j}$ the Joule heating rate. The figure shows a prominent, narrow layer co-spatial with the vortex structure seen in the previous figure. The Joule dissipation associated with the magnetic reconnection between the rising flux tube and the coronal magnetic field heats the plasma mostly in this region. 

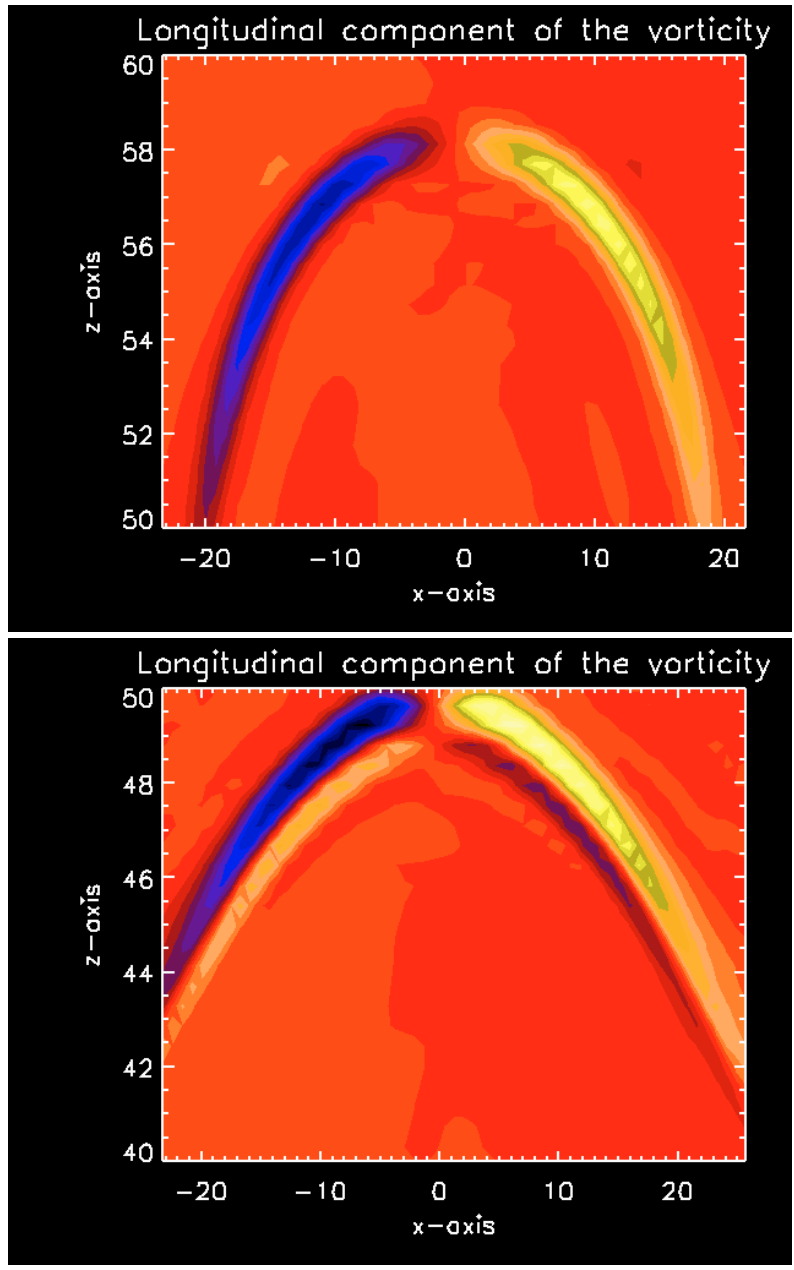

Fig. 11. The panels show the $y$-component of the vorticity in the $x z$ plane along the centre of the tube. Dark blue and yellow represents opposite, positive-negative, extrema of the vorticity. Top panel shows the case without a coronal magnetic field, while the bottom panel shows the case with a coronal magnetic field. The panels represent $t=64.7$ and $t=70.0$ respectively.

\section{Discussion and conclusions}

The problem of magnetic flux emergence from the solar convection zone through the photospheric region and into the corona has been undertaken. The numerical experiments presented consider a twisted magnetic flux tube lying in an adiabatically stratified interior. The initial disturbance is created by choosing a distribution of density along the tube axis so that the central regions are buoyant and start to rise toward the photosphere. Two classes of simulations have been undertaken.

Firstly, a series of simulations of a twisted flux tube emerging into a field-free corona have been performed. To optimize the link with previous attempts at this kind of experiment, we have used simplified initial conditions as found in the literature (Matsumoto et al. 1993, 1998; Magara \& Longcope 2001, 2003; Fan 2001), with, in particular, the buoyancy distribution along the tube chosen as in the paper by Fan. The aim of our simulations was to provide a physical understanding of various physical processes not mentioned (or not explained) in the previous literature. The initial stages of the rise

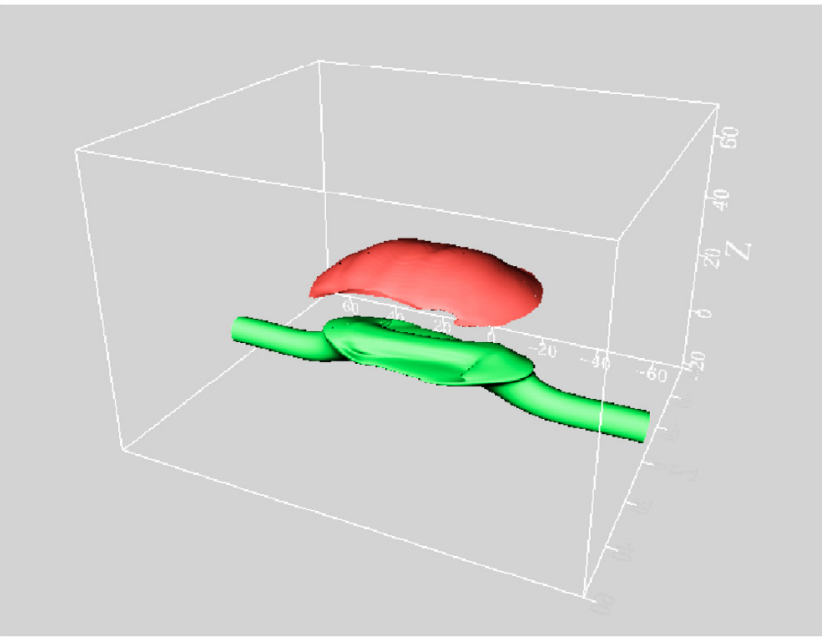

Fig. 12. An isosurface of $Q_{j} / \rho$ (red) and of constant magnetic field strenght (green) are shown. The red isosurface indicates the locations in space where the Joule dissipation has a significant impact on the heating of the plasma. Notice that the dome shaped structure outlining the region where the coronal magnetic field interacts with the rising flux tube $(t=80.5)$. The box represents the full size of the domain.

show a clear resemblance to the 2.5D tube rise calculations of Moreno-Insertis \& Emonet (1996). On the other hand, the initiation of the flux tube rise, due to magnetic buoyancy, generates a compressive wave that rapidly grows in amplitude and steepens to form a shock. We obtain excellent agreement with the results of Kalkofen et al. (1994) and Lamb (1932), which also serves as a further test of the numerical code, specially in view of the strong stratification in the background density. The emergence occurs in two distinct stages. The initial rise due to buoyancy slows down as the flux tube reaches the isothermally stratified photosphere: if unmagnetized, this very subadiabatic stratification would not allow the development of buoyant instabilities, due to its steep increase in entropy with height. In our case, though, the decrease of magnetic field intensity with height in the upper part of the rising tube provides a source of instability that is related (but different) to the Rayleigh-Taylor instability, as studied in detail in the literature of the 1960's and 1970's cited in Sect. 4.3. Instability ensues if the logarithmic magnetic pressure gradient is steeper than the normalized entropy gradient $c_{\mathrm{v}}^{-1} \mathrm{~d} S / \mathrm{d} r$ times the plasma $\beta$, with $c_{\mathrm{v}}$ the specific heat at constant volume. As the tube rises (even if slowly) in the photosphere, the plasma $\beta$ goes from well above 1 down to values below unity, and this allows the buoyant instability to develop: a runaway expansion occurs, the magnetized plasma rises and expands and it tries to fill the available coronal volume.

Pressure and density decrease steeply with height in the atmospheric regions below the corona (see, e.g., Fig. 1) and it is there that the rising plasma suffers the largest expansion. We note that the expansion takes place preferentially in the horizontal directions, so that the rising ball eventually adopts an oval, or flattened shape, with fast elongation in the $x$ and $y$ directions (Fig. 5). This process is accompanied by downflows in the surrounding plasma with speed comparable to those observed in the solar transition region and corona. Also because 
of the sideways expansion, the field line windings within the plasma ball (Fig. 7, right column) are increasingly inclined toward the flanks of the tube. This feature is akin to the fan-like shape seen in satellite (e.g., TRACE) coronal loop images. Also apparent in that same figure (top view panel), is that the horizontal projection of those field lines is almost perpendicular to the direction of the initial tube axis. This is a consequence of the high field line pitch in the tube at time $t=0$, and its increase following the expansion, as expected from elementary MHD considerations. Thus, the mutual orientation of coronal magnetic field and bipolar region in the surface cannot be easily matched with the corresponding solar observations, at least during these initial phases of the eruption into the atmosphere. A further consequence, discussed below, of the rapid quasiadiabatic expansion of the plasma in the atmosphere is that the temperature falls dramatically, in fact to values well below those in the initial stratification. Finally, the results of our simulations were used to produce synthetic magnetograms and dopplergrams. The appearance of the bipolar region on horizontal planes does depend on the height it is observed at. This is also linked to the twist profile of the rising flux tube.

A second class of simulations involved the emergence of the flux tube into a pre-existing horizontal coronal field. The orientation of the coronal field was selected to optimize the possibility of magnetic reconnection with the outermost flux tube field lines. When the upcoming and ambient coronal fields come into contact, a thin, dome-shaped current sheet is formed and reconnection is started, which changes the general topology of the magnetic field in the box: the reconnected field lines start as horizontal field lines of the coronal ambient field in the $x$-boundary, but end in the $y$-boundary as twisted field lines of the magnetic tube. The reconnection process continues by the pressing together of successive layers of upcoming plasma and ambient field. In fact, as time goes on, the reconnection extends to field lines situated sideways from the apex of the current sheet. The latter constitutes a genuine 3D effect, not present in simple 2D models. The reconnection process produces plasma heating due to Joule dissipation, but the consequent rise in temperature is limited to just the region close to the reconnection site.

Some of the features listed in the foregoing paragraphs need some further discussion. The initial conditions for the magnetic field distribution used in the current paper (as well as in basically all related literature) provide for a fast emergence to the photosphere, which is a pre-condition for the feasibility of this type of 3D numerical experiments. Choosing this kind of initial condition, though, is not strictly in agreement with what we know about the evolution of the magnetic field in the convection zone. From general physical reasoning as well as through the results of the experiments on the emergence of magnetic tubes across the convection zone, we expect the rising tubes in the final few Megametres of their rise below the photosphere to have (1) large rising speeds (say, up to order one tenth the local sound speed); (2) a plasma beta clearly above unity; and (3) an entropy which corresponds to that of their initial location, since the rise in the convection zone should be faster than the local timescales for thermal exchange and, hence, basically adiabatic. Condition (2), in particular, means that there is no compelling argument to assume a force-free situation for the initial tube. The choice of entropy distribution, on the other hand, is not trivial, since it influences the buoyancy distribution and, hence, the rise of the tube to the photosphere. In Sect. 3.2 we showed how the standard choices in the literature of $3 \mathrm{D}$ flux emergence do not really fit with either a deep origin of the magnetic flux at the bottom of the convection zone nor a local assembling of the flux by, e.g., the supergranules (to produce, say, small ephemeral active regions). So, the initial condition chosen and its implications on the emergence process should be subjected to detailed study in the future.

One of the major limitations of the 3D simulations of emergence into the atmosphere so far is the lack of an adequate description of the entropy sources in the energy equation (e.g., radiative transfer in photosphere and chromosphere, or, from a different perspective, direct energy deposition in chromosphere and corona via e.g. wave dissipation). This fact combined with the large expansion of the plasma when rising above the photosphere without explicit sources of chromospheric or coronal heating leads to low temperatures in the magnetized volume in those levels, well below what can be expected in the real Sun. Now, in the Sun the chromosphere is expected to have a two-component structure, with cold and hot regions simultaneously present, the latter being created by the dissipation of vertically running waves generated mainly through the ongoing convection in the (sub)photospheric levels and steepening to yield shocks (Ayres 1981; Asensio Ramos et al. 2003; Carlsson \& Stein 2002; Wedemeyer et al. 2004). On the other hand, the corona is expected to be heated through nanoflares or, perhaps, also wave dissipation (see, e.g., Walsh 2002). Our code does not cope with all those mechanisms, and including them at the present stage would unduly complicate the simplicity of the current numerical experiments. Yet, even without extra entropy sources, the experiments presented already show results which resemble features observed in the Sun. As a simple alternative, one can use an ad-hoc heating/cooling term directly proportional to the deviation from the initial temperature profile (Newton's law), as used by Abbett \& Fisher (2003). Yet, even at the price of obtaining unrealistic temperatures, in the present context it is better to carry out simulations with as simple thermodynamics as possible, to spot the fundamental MHD effects, leaving the inclusion of additional ingredients for future papers. Nonetheless, in the present simulation there is ohmic and viscous dissipation included via a hyperdiffusive ansatz, which are important basically at interfaces and regions of sharp magnetic field gradients. In fact, in the second set of simulations (Sect. 7), we observe important heating in the thin current sheet formed in the interface between upcoming plasma and ambient coronal field. This heating being limited basically to a shell (Fig. 12), can not heat the volume of rising magnetized plasma below.

As a counterpart, numerical dissipation associated with the limitations on spatial resolution can provide an unwanted source of entropy which can importantly change the results of the experiments. A case in point has to do with the buoyancy in the convection zone. The initial magnetic structure is necessarily concentrated. Choosing too coarse a resolution for the volume occupied by the initial tube leads to ohmic 
dissipation, which artificially increases the entropy in the initial stages of the rise. This leads to an increase of temperature, decrease of density and, from there, enhanced buoyancy in the tube. Now, the dynamics of the initial phase is typically driven by buoyancy with a density deficit of order the canonical Parker value, $|\Delta \rho / \rho| \sim 1 / \beta$. In a high- $\beta$ environment, one has to take extra care that the density deficit is not unduly increased through numerical dissipation. This puts a strict lower limit to the resolution to be used in mapping the convection zone in the experiment. It is perhaps of interest to note that one cannot assign a single value of the viscous and magnetic Reynolds number as such for the whole experiment. Values for Re can be determined after the run, assuming that the Joule dissipation is simply given by $\eta j^{2}$. Doing this, one can obtain mean values in the different regions varying between 100 and several thousand. Similar difficulties apply when trying to define a global Lundquist number.

This paper represents a first attempt at a detailed description of the process of eruption of magnetic field into the atmosphere. The parameters chosen correspond to a small active region or a standard ephemeral active region. To keep the physics simple (and, hence, to maximize the possibilities of analyzing and understanding the results), we have used drastic simplifications of the initial stratification and of the thermodynamics of the plasma, not allowing for preexisting convection below the photosphere, radiative transfer (which is important especially in the low atmosphere), nor heat conduction or explicit radiative cooling (of importance in the corona), in line with previous similar attempts in the literature. Future work will have to incorporate gradually those additional features.

Acknowledgements. Computational time on the following massively parallel computers is gratefully acknowledged: SRIF and PPARC funded Linux cluster in St. Andrews (Scotland, UK); SGI Origin 2000 in CIEMAT (Madrid, Spain); MCYT and IAC funded Linux Cluster in La Laguna (Tenerife, Spain); PPARC funded Compaq MHD Cluster (St Andrews, UK); UK Astrophysical Fluids Facility (UKAFF). This work has also benefited from financial support through the Platon European Research Training Network HPRN-CT-2000-00153 of the European Commission and the CICYT project No. AYA2001-1649 of the Spanish Ministery of Science and Technology (MCYT). KG was supported by PPARC in the form of an Advanced Fellowship and by the Carlsberg Foundation in the form of a fellowship.

\section{References}

Abbett, W. P., \& Fisher, G. H. 2003, ApJ, 582, 475

Acheson, D. J. 1979, Sol. Phys., 62, 23

Asensio Ramos, A., Trujillo Bueno, J., Carlsson, M., \& Cernicharo, J. 2003, ApJ, 588, 61L

Ayres, T. R. 1981, ApJ, 244, 1064

Brekke, P., Hassler, D. M., \& Wilhelm, K. 1997, Sol. Phys., 175, 349 Caligari, P., Moreno-Insertis, F., \& Schüssler, M. 1995, ApJ, 441, 886 Caligari, P., Schüssler, M., \& Moreno-Insertis, F. 1998, ApJ, 502, 481 Carlsson, M., \& Stein, R. F. 1992, ApJ, 397, 59
Carlsson, M., \& Stein, R. F. 2002, ApJ, 572, 626

Cattaneo, F., Chiueh, T., \& Hughes, D. W. 1990, J. Fl. Mech., 219, 1

Chae, J., Wang, H., Qiu, J., Goode, P., \& Wilhelm, K. 2000, ApJ, 533, 535

Choudhuri, A. R. 1987, ApJ, 316, 788

Dorch, S. B. F., Archontis, V., \& Nordlund, A. 1999, A\&A, 352, 79

D'Silva, S., \& Choudhuri, A. R. 1993, A\&A, 272, 621

Emonet, T., \& Moreno-Insertis, F. 1998, ApJ, 492, 804

Fan, Y. 2001, ApJ, 554, L111

Fan, Y., \& Gibson, S. E. 2003, ApJ, 589, L105

Fan, Y., Fisher, G. H., \& McClymont, A. N. 1994, ApJ, 436, 907

Fan, Y., Zweibel, E. G., Linton, M. G., \& Fisher, G. H. 1998, ApJ, 505, L59

Hughes, D. W., \& Proctor, M. R. E. 1988, Ann. Rev. Fluid Mech, 20, 187

Kalkofen, W., Rossi, P., Bodo, G., \& Massaglia, S. 1994, A\&A, 284, 976

Krall, J., Chen, J., Santoro, R., et al. 1998, ApJ, 500, 992

Kusano, K., Moriyama, K., \& Miyoshi, T. 1998, Phys. Plasmas, 5, 2582

Lamb, H. 1932, Hydrodynamics (New York: Dover)

Magara, T. 2001, ApJ, 549, 608

Magara, T., \& Longcope, D. W. 2001, ApJ, 559, 55

Magara, T., \& Longcope, D. W. 2003, ApJ, 586, 630

Manchester, W. 2001, ApJ, 547, 503

Matsumoto, R., \& Shibata, K. 1992, PASJ, 44, 167

Matsumoto, R., Tajima, T., Shibata, K., \& Kaisig, M. 1993, ApJ, 414, 357

Matsumoto, R., Tajima, T., Chou, W., Okubo, A., \& Shibata, K. 1998, ApJ, 493, L43

Moreno-Insertis, F. 1986, A\&A, 166, 291

Moreno-Insertis, F. 1997, Mem. Soc. Astr. It., 68, 429

Moreno-Insertis, F. 2004, in preparation

Moreno-Insertis, F., \& Emonet, T. 1996, ApJ, 472, L53

Moreno-Insertis, F., Caligari, P., \& Schüssler, M. 1994, Sol. Phys., 153, 449

Newcomb, W. A. 1961, Phys. Fl., 4, 391

Nordlund, A., \& Galsgaard, K. 1997, Tech. Rep., Astronomical Observatory, Copenhagen University

Nozawa, S., Shibata, K., Matsumoto, R., et al. 1992, ApJS, 78, 267

Parker, E. N. 1975, ApJ, 198, 205

Priest, E., \& Forbes, T. 2000, Magnetic Reconnection MHD Theory and Applications (Cambridge Univ. Press)

Priest, E., Titov, V., Vekestein, G., \& Rickard, G. 1994, J. Geophys. Res., 99, A11, 21

Shibata, K., Tajima, T., Matsumoto, R., et al. 1989, ApJ, 338, 471

Shibata, K., Nozawa, S., \& Matsumoto, R. 1992, PASJ, 44, 256

Sonnerup, B. U. Ö. 1988, Comp. Phys. Commun., 49, 142

Spruit, H. C. 1977, Ph.D. Thesis, Univ. Utrecht

Spruit, H. C., \& van Ballegooijen, A. A. 1982, A\&A, 106, 58

Spruit, H. C., Title, A., \& van Ballegooijen, A. A. 1987, Sol. Phys., 110,115

Thomas, J. H., \& Nye, A. H. 1975, Phys. Fl., 18, 490

Walsh, R. W. 2002, in From Solar Min to Max: Half a solar cycle with SOHO, ESA SP-508, 253

Wedemeyer, S., Freytag, B., Steffen, M., Ludwig, H.-G., \& Holweger, H. 2004, A\&A, 414, 1121

Yu, C. P. 1965, Phys. Fl., 8, 650 